\title{
SOME PROBLEMS AND TECHNIQUES IN SET-THEORETIC TOPOLOGY
}

\author{
FRANKLIN D. TALL ${ }^{1}$
}

\begin{abstract}
I survey some problems and techniques that have interested me over the years, e.g. normality vs. collectionwise normality, reflection, preservation by forcing, forcing with Souslin trees, and Lindelöf problems.
\end{abstract}

\section{INTRODUCTION}

In 2008-9 I gave a graduate course in set-theoretic topology at the University of Toronto. With retirement on the horizon, I wanted to pass on to the next generation some of the topological problems I had found interesting and some of the set-theoretic techniques I had found useful over my 40 years of research. Marion Scheepers - one of the editors of this volume - asked me to send him copies of the lecture notes my students prepared. He then suggested I make them into an article for this volume. I was initially dubious, since most of the material had been published elsewhere. I concluded, however, that I had indeed added enough value to the extracted material to make the project worthwhile. In particular, I had included lots of "what's important" and "what's really going on" commentary often missing from journal articles. I also realized that these notes could serve as an update of my 1984 survey Normality versus collectionwise normality [Tal84], as well as call attention to some interesting work over the past few years on forcing with Souslin trees, the publication of which has been indefinitely delayed. The intended audience, then, is composed of graduate students interested in set-theoretic topology, topologists interested in learning some useful set-theoretic techniques scattered through the literature, and set theorists interested in applications of set theory to topological problems. Prerequisites include a basic knowledge of general topology, and a first graduate course in set theory. For the former, I recommend Engelking [Eng89]; for the latter, Kunen [Kun80].

\section{Normality versus COllectionwise Hausdorffness}

The relationship between normality and collectionwise Hausdorffness (every closed discrete subspace can be separated, i.e. expanded to a disjoint collection of open sets, each containing only one of the points of the subspace) was one of the first areas in general topology to feel the impact of set-theoretic methods [Tal77]. [Tal84]

2010 Mathematics Subject Classification. Primary 54A35, 54D10, 54D20, 54D45; Secondary $54 \mathrm{D} 50$.

Key words and phrases. Normal, collectionwise Hausdorff, collectionwise normal, first countable, locally compact, Cohen real, random real, forcing, $\sigma$-centered, supercompact, reflection, preservation under forcing, elementary submodel, irrationals, forcing with a Souslin tree, Lindelöf.

${ }^{1}$ The author was supported in part by NSERC Grant A-7354. 
exhaustively surveys the subject up to the time of its writing. Since then, there have been several interesting developments.

The question of whether locally compact normal metacompact spaces were paracompact had been raised by Arhangel'skil and by the author [Arh72], [Tal74]. [Wat82] showed that $V=L$ yielded an affirmative answer. By a difficult forcing argument, a consistent counterexample was constructed by Gruenhage and Koszmider [GK96a]. It had been known since the 1960's that collectionwise Hausdorffness sufficed to make such spaces paracompact; in [GK96b], Gruenhage and Koszmider showed that $\aleph_{1}$-collectionwise Hausdorffness sufficed in the course of their surprising proof that:

Proposition 1.1. $M A_{\omega_{1}}$ implies locally compact normal metalindelöf spaces are paracompact.

It had been known for a long time that Katětov's problem: are compact spaces with hereditarily normal squares metrizable? ("Yes" for cubes [Kat48]) could be solved if one combined "S \& L" consequences of $M A_{\omega_{1}}$ with the non-existence of separable normal first countable spaces which are not collectionwise Hausdorff. The solution of this problem by Larson and Todorcevic [LTo02] opened a new front in the study of normality versus collectionwise normality. The question became, could other long-standing open problems be solved by consistently combining consequences of $M A_{\omega_{1}}$ or PFA with "normality implies collectionwise Hausdorff" consequences of $V=L$ ? There has been one notable success here, which we shall get into shortly, and a number of interesting open problems. We will start off with a result that no one had even conjectured before [LTaa], but in retrospect seems a plausible variation on the following classical theorem [Tal77], [Tal84], [Tal88].

Proposition 1.2. Adjoin $\aleph_{2}$ Cohen subsets of $\omega_{1}$. Then normal spaces of character $\leq \aleph_{1}$ are $\aleph_{1}$-collectionwise Hausdorff.

The newer result is:

Theorem 1.3 ([LTaa]). Force with a Souslin tree. Then normal first countable spaces are $\aleph_{1}$-collectionwise Hausdorff.

The proof uses ideas from both Fleissner's $\diamond$-for-stationary-systems proof [Fle74] and from the Cohen-subsets-of- $\omega_{1}$ proof. These are both included in my 1984 survey. From the former, construct a partition that ruins all normalizations, and from the latter, arrange to successively decide initial segments of potential normalizations. First countability, the countable chain condition, and $\omega$-distributivity ensure that closed unboundedly often, one can decide $f \mid \alpha$, for $f \in \omega^{\omega_{1}} \omega$ and $\alpha \in \omega_{1}$. It is not known whether Theorem 1.3 can be improved to "character $\leq \aleph_{1}$ ". Now for the details.

Lemma 1.4. After forcing with a Souslin tree, the following holds. Suppose that $\left\{N(\alpha, i): i<\omega, \alpha<\omega_{1}\right\}$ are sets such that for all $\alpha, i, N(\alpha, i+1) \subseteq N(\alpha, i)$. Suppose further that for all $A \subseteq \omega_{1}$, there is an $f: \omega_{1} \rightarrow \omega$ such that:

$$
\bigcup\{N(\alpha, f(\alpha)): \alpha \in A\} \cap \bigcup\left\{N(\beta, f(\beta)): \beta \in \omega_{1} \backslash A\right\}=\emptyset .
$$

Then there is a $g: \omega_{1} \rightarrow \omega$ and a closed unbounded $C \subseteq \omega_{1}$, such that:

$$
\text { whenever } \alpha<\beta \text { and } C \cap(\alpha, \beta] \neq \emptyset \text {, then } N(\alpha, g(\alpha)) \cap N(\beta, g(\beta))=\emptyset \text {. }
$$


It should be clear that Lemma 1.4 yields $\aleph_{1}$-collectionwise Hausdorffness in first countable normal spaces: let the $N(\alpha, i)$ 's be a descending neighborhood base at $\alpha$, where we have labeled the points of a discrete closed subspace of the space $X$ with the countable ordinals. Let $g$ and $C$ be as in the statement of the Lemma. Define $c: \omega_{1} \rightarrow \omega_{1}$ by letting $c(\alpha)=\sup ((C \backslash\{0\}) \cap(\alpha+1))$, and let $\alpha \sim \beta$ if $c(\alpha)=c(\beta)$. The $\sim$-classes are countable and normality implies $\aleph_{0}$-collectionwise Hausdorffness, so there is a $q: \omega_{1} \rightarrow \omega$ such that $c(\alpha)=c(\beta)$ implies $N(\alpha, q(\alpha)) \cap N(\beta, q(\beta))=\emptyset$. Let $r(\alpha)=\max (g(\alpha), q(\alpha))$. Then $\left\{N(\alpha, r(\alpha)\}_{\alpha<\omega_{1}}\right.$ is the required separation.

Proof of Lemma 1.4. Let $S$ be a Souslin tree. Let $\left\{\dot{N}(\alpha, i): i<\omega, \alpha<\omega_{1}\right\}$ be $S$-names as in the hypothesis. For $s \in S$, let $\ell(s)$ be the length of $s$. Since $S$ has countable levels and its corresponding forcing poset is $\omega$-distributive, we can construct an increasing function $h: \omega_{1} \rightarrow \omega_{1}$ such that for all $\alpha<\omega_{1}$ and all $s \in S$ with $\ell(s)=h(\alpha)$, s decides all statements of the form " $\dot{N}(\beta, j) \cap \dot{N}(\alpha, i)=\emptyset$ ", for all $i, j<\omega$ and $\beta<\alpha$.

Let $\dot{A}$ be an $S$-name for a subset of $\omega_{1}$ such that for no $\alpha<\omega_{1}$ does any $s \in S$ with $\ell(s)=h(\alpha)$ decide whether $\alpha \in A$. To define such an $\dot{A}$, for each $\alpha<\omega_{1}$ pick two successors of each $s \in S$ with $\ell(s)=h(\alpha)$ and let one force $\alpha \in \dot{A}$ and let the other force $\alpha \notin \dot{A}$.

Let $\dot{f}$ be an $S$-name for a function $f: \omega_{1} \rightarrow \omega$ as in the hypothesis of the lemma, with respect to $A$. Let $C$ be a closed unbounded subset of $\omega_{1}$ in $V$ such that for each $s \in S$ with $\ell(s) \in C, s$ decides $\dot{f} \mid \ell(s)$ and $\dot{A} \mid \ell(s)$, and such that for all $\alpha<\beta<\omega_{1}$, if $\beta \in C$ then $h(\alpha)<\beta$. We will define an $S$-name $\dot{g}$ for a function from $\omega_{1}$ to $\omega$ such that whenever $\alpha<\beta<\omega_{1}$, if $(\alpha, \beta] \cap C \neq \emptyset$, then $N(\alpha, g(\alpha)) \cap N(\beta, g(\beta))=\emptyset$.

Let $c: \omega_{1} \rightarrow \omega_{1}$ be defined as above. Then for all $\beta<\alpha<\omega_{1}, c(\alpha)=c(\beta)$ if and only if $C \cap(\alpha \backslash \beta)=\emptyset$. Fix $\beta<\omega_{1}$. Each $s \in S$ with $\ell(s)=h(\beta)$ decides $\dot{f} \mid c(\beta)$ and $A \mid c(\beta)$ and " $\dot{N}(\alpha, \dot{f}(\alpha)) \cap \dot{N}(\beta, i)=\emptyset$ " for all $i<\omega, \alpha<c(\beta)$, but not whether $\beta \in A$. Fix $s \in S$ with $\ell(s)=h(\beta)$. Since $s$ does not decide whether $\beta \in A$, we claim that there is an $i_{0}<\omega$ such that for all $\alpha<c(\beta)$ such that $s \Vdash \alpha \in \dot{A}$, $s \Vdash \dot{N}(\alpha, \dot{f}(\alpha)) \cap \dot{N}\left(\beta, i_{0}\right)=\emptyset$.

To see this, extend $s$ to $t \in S$ forcing that $\beta \notin A$ and deciding $f(\beta)$. Let $i_{0}$ be the value of $f(\beta)$ as decided by $t$. Then for each $\alpha<c(\beta)$ such that $s \Vdash \alpha \in \dot{A}$, $t$ forces that $N(\alpha, f(\alpha)) \cap N\left(\beta, i_{0}\right)=\emptyset$, but these facts were already decided by $s$. Similarly, there is an $i_{1}<\omega$ such that for all $\alpha<c(\beta)$ such that $s \Vdash \alpha \notin \dot{A}$, $s \Vdash \dot{N}(\alpha, \dot{f}(\alpha)) \cap \dot{N}\left(\beta, \dot{i}_{1}\right)=\emptyset$.

Since $s$ decides $A \mid c(\beta)$, letting $\bar{i}=\max \left\{i_{0}, i_{1}\right\}$, for all $\alpha<c(\beta), s \Vdash \dot{N}(\alpha, \dot{f}(\alpha)) \cap$ $\dot{N}(\beta, \bar{i}))=\emptyset$.

We have such an $\bar{i}_{s}$ for each $s$ in the $c(\beta)$-th level of the tree, so we can construct a name $\dot{g}$ such that $s \Vdash \dot{g}(\beta)=\max \left\{\bar{i}_{s}, \dot{f}(\beta)\right\}$ for each $s \in S$ with $\ell(s)=c(\beta)$. Then $\dot{g}$ is as required.

It is not clear how Theorem 1.3 fits into the panoply of "normal implies collectionwise normal" results. Because the proof doesn't obviously generalize to character $\leq \aleph_{1}$, the standard technique [Wat82] for obtaining "locally compact normal implies $\aleph_{1}$-collectionwise Hausdorff" is unavailable. However, one desired extension does go through without difficulty, Although Souslin tree forcing does not make normal first countable spaces collectionwise Hausdorff [Tal11c], one can arrange for that statement to hold in the models one is likely to be interested in, simply by 
starting with a nice enough ground model. In particular, one can start with a model of $G C H$, adjoin $\lambda^{+}$Cohen subsets of $\lambda$ for each regular $\lambda$ via an Easton extension [Eas70], [Tal88], establishing $\diamond$ for stationary systems for all such $\lambda$. Forcing over this model with an $\aleph_{2}$-chain condition partial order $\mathbb{P}$ of size $\aleph_{2}$ will preserve $\diamond$ for stationary systems at $\aleph_{2}$ and above. Thus, if, say, $\mathbb{Q}=\mathbb{P} \times \mathbb{S}$, where $\mathbb{S}$ is a Souslin tree, in the resulting model normal first countable spaces will be collectionwise Hausdorff.

This observation is quite interesting from the point of view of obtaining paracompactness. It is well-known that paracompactness is equivalent to subparacompactness (every open cover has a $\sigma$-discrete closed refinement) plus collectionwise normality: expand a $\sigma$-discrete closed refinement to a $\sigma$-discrete open refinement. If the space is e.g. locally compact, one can get away with collectionwise normality for compact sets or even (this is not obvious) collectionwise Hausdorffness. With this in mind, models in which one can easily obtain $\sigma$-discrete closed refinements as well as obtaining "normal implies collectionwise Hausdorff" results are quite promising. In 1983, Balogh [Bal83] proved under $M A_{\omega_{1}}$ that locally countable subsets of size $\aleph_{1}$ in a compact countably tight space are $\sigma$-discrete. Under suitable hypotheses, this can be improved to $\sigma$-closed-discrete, and the paracompactness idea mentioned above comes into play, if one assumes or proves collectionwise Hausdorffness. Balogh assumed collectionwise Hausdorffness in [Bal83] and [Bal02], as did Nyikos in a number of papers on hereditarily normal manifolds, e.g. [Nyi02]. What one would like to do is obtain Balogh's $M A_{\omega_{1}}$ result in one of the models obtained by forcing with a Souslin a tree. The assumption of "collectionwise Hausdorff" could then likely be removed, since normality was assumed anyway. We will comment further on Balogh's result shortly, but we now see the interest in retaining consequences of $M A_{\omega_{1}}$ (or PFA, etc.) when forcing with a Souslin tree.

Definition 1.5. A space is $\aleph_{1}$-collectionwise Hausdorff if each closed discrete subspace $D$ of size $\aleph_{1}$ can be separated by disjoint open sets. It is weakly $\aleph_{1}$ collectionwise Hausdorff if $\aleph_{1}$ of the points of $D$ can always be separated. It is stationarily $\aleph_{1}$-collectionwise Hausdorff if stationarily many can be separated for any well-ordering of $D$. It is very stationarily $\aleph_{1}$-collectionwise Hausdorff if stationarily many of the points of any stationary subset of $D$ can be separated, for any well-ordering of $D$. closed unboundedly $\aleph_{1}$-collectionwise Hausdorff is defined analogously to "stationarily $\aleph_{1}$-collectionwise Hausdorff".

Proposition 1.6 ([Tay81]). Closed unboundedly $\aleph_{1}$-collectionwise Hausdorff spaces are $\aleph_{1}$-collectionwise Hausdorff.

Proposition 1.7. $2^{\aleph_{0}}<2^{\aleph_{1}}$ implies that normal spaces of character $\leq 2^{\aleph_{0}}$ are stationarily $\aleph_{1}$-collectionwise Hausdorff [Tay81] and hence locally compact normal spaces are stationarily $\aleph_{1}$-collectionwise Hausdorff [Tal84].

There is an example consistent with $G C H$ of a first countable, locally compact normal space which is stationarily $\aleph_{1}$-collectionwise Hausdorff, but not very stationarily $\aleph_{1}$-collectionwise Hausdorff [DS79], [Tal84].

Problem 1.8. Is every locally compact, normal, very stationarily $\aleph_{1}$-collectionwise Hausdorff space $\aleph_{1}$-collectionwise Hausdorff?

The proof of Gruenhage and Koszmider actually establishes that: 
Proposition 1.9. Locally compact, normal, metalindelöf, very stationarily $\aleph_{1-}$ collectionwise Hausdorff spaces are paracompact.

It is an interesting and largely unexplored question as to what one can get by just forcing with a Souslin tree. See [LTo01], [LTo02], [Tod]. It turns out that by forcing with a particular kind of Souslin tree, over particular models, one can get quite a lot.

\section{Forcing With COHEREnt Souslin trees}

Definition 2.1. A coherent tree is a downward closed subtree $S$ of $<\omega_{1} \omega$ with the property that $\{\vec{\xi} \in \operatorname{dom} s \cap \operatorname{dom} t: s(\vec{\xi}) \neq t(\vec{\xi})\}$ is finite for all $s, t \in S$. A coherent Souslin tree is a Souslin tree given by a coherent family of functions in $<\omega_{1} \omega$ closed under finite modifications.

Coherent Souslin trees can be constructed from $\diamond$ [Lar99].

Theorem 2.2 ([LTo02]). Let $S$ be a coherent Souslin tree. Iterate countable chain condition posets that preserve $S$ to obtain $M A_{\omega_{1}}$ restricted to such posets. Then force with $S$. In the resulting model, compact spaces with hereditarily normal squares are metrizable.

The most interesting models to force over with coherent Souslin trees are models of iteration axioms such as $M A_{\omega_{1}}, P F A$, and Martin's Maximum, restricted to posets that preserve the "Souslinity" of the tree. A good many of the consequences of these axioms survive the additional forcing, but whether some others do are hard open questions we shall mention later.

Definition 2.3. Let $S$ be a coherent Souslin tree. $P F A(S)$ is PFA restricted to proper posets that preserve $S$. Let $\Phi$ be a proposition. $P F A(S)[S] \Vdash \Phi$ is an abbreviation for " $\Phi$ holds in any model obtained by forcing with $S$ over a model of PFA(S)". $M A_{\omega_{1}}(S)[S], M M(S)[S]$ are similarly defined.

$P F A(S)[S]$ is not an axiom, but is convenient to act as if it were. Proofs involving $P F A(S)[S]$ are similar to, but more difficult than, those for $P F A$, since one tries to apply $P F A(S)$ to a partial order involving $S$-names. The intuition behind the use of coherence is that when forcing with a coherent Souslin tree, two different interpretations of an $S$-name will essentially be isomorphic, since, up to an automorphism of $S, S$ has only one generic branch. It is unfortunate that the fundamental work of Todorcevic concerning forcing with a coherent Souslin tree over a model of $P F A(S)$ has not been published. It is hoped that [Tod] will eventually appear.

Of course if $P F A(S)[S]$ only provided harder proofs of $P F A$ consequences, that would not be very interesting. As we shall see, that trick is to combine those consequences with consequences of forcing with $S$.

The most useful consequence of $P F A(S)[S]$ from the point of view of obtaining paracompactness is Balogh's

$\sum$ : Suppose $X$ is a compact, countably tight space and $Y$ is a subset of $X$ of size $\aleph_{1}$ such that for each $y \in Y$ there exist open sets $U_{y}, V_{y}$ such that $y \in V_{y} \subseteq \bar{V}_{y} \subseteq U_{y}$, and $\left|U_{y} \cap Y\right|$ is countable. Then $Y=\bigcup_{n<\omega} Y_{n}$, where each $Y_{n}$ is closed discrete in $\bigcup\left\{V_{y}: y \in\right.$ $Y\}$. 
It is not hard to show that $\sum$ implies there are no compact $S$-spaces. $\sum$ was obtained from $M A_{\omega_{1}}$ in [Bal83]. If the space has Lindelöf number $\leq \aleph_{1}$, we may assume the $Y_{n}$ 's are closed discrete in the whole space. Todorcevic [unpublished] proved this from $P F A(S)[S]$ in 2002. A detailed proof of the special case in which finite products of $S$ are sequential is supposed to appear in [Fis]. An essential ingredient in Todorcevic's proof is:

Theorem 2.4. PFA(S)[S] implies compact countably tight spaces are sequential.

The compact $S$-space proof and the proof of Theorem 2.4 were sketched by Todorcevic in his talk at Erice in 2008 [Tod08].

The reader may have some idea as to how normality plus collectionwise Hausdorffness plus $\sum$ could yield a $\sigma$-discrete open refinement of an open cover of size $\aleph_{1}$, but what about open covers of other sizes? The answer is that in a major case of interest, those are all one needs to be concerned about.

Definition 2.5. Axiom $R$ [Fle86] is the assertion that if $\mathcal{S}$ is a stationary subset of $[\kappa]^{\omega}$ and $\mathcal{C}$ is a subset of $[\kappa]^{\omega_{1}}$ closed under unions of strictly increasing $\omega_{1}$-chains, then there is a $Y \in \mathcal{C}$ such that $\mathcal{S} \cap[Y]^{\omega}$ is stationary.

Lemma 2.6 ([Bal02]). Axiom $R$ implies that if $X$ is a locally Lindelöf, regular, countably tight space such that every Lindelöf $Y \subseteq X$ has Lindelöf closure, then if $X$ is not paracompact, it has a clopen non-paracompact subspace with Lindelöf number $\aleph_{1}$.

Although Axiom $R$ does not follow from $P F A(S)[S]$ [Tal11c], it holds in the natural iteration model for $\operatorname{PFA}(S)[S]$ described above [LTab].

Reducing the question of paracompactness to subspaces with Lindelöf number $\aleph_{1}$ in countably tight spaces in which Lindelöf spaces have Lindelöf closures allows a further simplification.

Definition 2.7 ([Nyi84]). A space $X$ is of Type I if $X=\bigcup_{\alpha<\omega_{1}} X_{\alpha}$, where each $X_{\alpha}$ is open, $\alpha<\beta$ implies $\bar{X}_{\alpha} \subseteq \bar{X}_{\beta}$, and each $\bar{X}_{\alpha}$ is Lindelöf. $\left\{X_{\alpha}: \alpha<\omega_{1}\right\}$ is canonical if for limit $\alpha, X_{\alpha}=\bigcup_{\beta<\alpha} X_{\beta}$.

Lemma 2.8 ([EN09]). If $X$ is locally compact with Lindelö number $\leq \aleph_{1}$, and each Lindelöf subspace of $X$ has Lindelöf closure, then $X$ is of Type I, with a canonical sequence.

Lemma 2.9 ([Nyi84]). If $X$ is of Type $I$, then $X$ is paracompact if and only if $\left\{\alpha: \bar{X}_{\alpha} \backslash X_{\alpha} \neq 0\right\}$ is non-stationary.

Theorem 2.10. There is a model of PFA(S)[S] in which every $\aleph_{1}$-collectionwise Hausdorff, locally compact normal space in which closures of Lindelöf subspaces are Lindelöf, and which does not include a perfect preimage of $\omega_{1}$, is paracompact.

Proof. We may confine ourselves to Type I spaces. If $X$ is not paracompact, we may pick a canonical sequence and a stationary $S \subseteq \omega_{1}$ and $x_{\alpha} \in \bar{X}_{\alpha} \backslash X_{\alpha}$, for each $\alpha \in S . S$ is $\sigma$-closed-discrete because of:

Lemma 2.11 ([Bal83]). The one-point compactification of a locally compact space $X$ is countably tight if and only if $X$ does not include a perfect pre-image of $\omega_{1}$.

By normality and collectionwise Hausdorffness, we can find a stationary set $S^{\prime} \subseteq$ $S$ of limit ordinals such that $\left\{x_{\alpha}: \alpha \in S^{\prime}\right\}$ is closed discrete. By normality and 
collectionwise Hausdorffness, we can expand the $x_{\alpha}$ 's to a discrete collection of open $U_{\alpha}$ 's. Each $U_{\alpha}$ intersects some $X_{\beta}, \beta<\alpha$; pressing down yields an uncountable closed discrete subspace of some $\bar{X}_{\beta}$, contradiction.

I believe the " $\aleph_{1}$-collectionwise Hausdorff" hypothesis is superfluous, but I have not yet finished proving that.

Corollary 2.12 ([LTaa]). There is a model of PFA(S)[S] in which every locally compact, perfectly normal space is paracompact.

Proof. It is easy to see that a perfectly normal space cannot include a perfect preimage of $\omega_{1}$. A locally compact, perfectly normal space is first countable. It thus suffices to show that Lindelöf subspaces have Lindelöf closures. We use an idea from [Nyi03]. In fact, it suffices to show that open Lindelöf subspaces do, since by local compactness, such a subspace $Y$ can be covered by countably many open Lindelöf sets. The closure of that union is then Lindelöf and includes $\bar{Y}$. Let then $B$ be a right-separated subspace of the boundary of an open Lindelöf subspace $Y$. We claim $B$ is countable, whence the boundary of $Y$ is hereditarily Lindelöf. If $B$ is uncountable, it has an uncountable closed discrete subspace. Via normality and $\aleph_{1}$-collectionwise Hausdorffness, we can expand this to a discrete collection of open sets, giving a contradiction.

It is very much an open question what other consequences of $P F A$ hold under $P F A(S)[S]$ (or $M M(S)[S]$, or $M A_{\omega_{1}}(S)[S]$ ). Three of the most intriguing problems are:

(1) Does $P F A(S)[S]$ imply every hereditarily separable $T_{3}$ space is hereditarily Lindelöf?

(2) Does $M M(S)[S]$ imply first countable perfect pre-images of $\omega_{1}$ must include a copy of $\omega_{1}$ ?

(3) Is there a model of $P F A(S)[S]$ in which locally compact normal spaces are collectionwise Hausdorff?

The first is very natural, considering that $P F A$ implies the conclusion, and that $P F A(S)[S]$ implies there are no such compact spaces. The answer is not known even if the additional assumption of first countability is made.

The second would solve a longstanding open problem of Nyikos [Nyi83]: I have shown that i) the conclusion of (2) plus ii) a form of P-ideal dichotomy [AT97] plus iii) $\sum$ plus iv) normal first countable spaces are $\aleph_{1}$-collectionwise Hausdorff implies hereditarily normal manifolds of dimension $>1$ are metrizable. All except i) are known to follow from $P F A(S)[S]$ [Tod], [Fis], [LTaa]. Todorcevic proposed $M M(S)[S]$ rather than $P F A(S)[S]$ since a proof may involve shooting a club through a stationary set.

The third has been annoying me for several years; I thought I had a proof, but there was a gap which I hope to finish filling in the near future. It is natural to expect this to be true, since any other general theorem about when normality implies some form of collectionwise normality if true about first countable spaces seems to turn out to be true about locally compact spaces. [LTab], [Tal11a], [Tal11c] explore the consequences of an afirmative answer.

I had originally planned to put in a $P F A(S)[S]$ proof into this survey, but in fact there have been no very successful applications so far of Todorcevic's methods other than those he has done, none of which he has published. (The $M A_{\omega_{1}}(S)[S]$ 
technique is exhibited in [LTo02].) Such a proof would also add at least 10 pages to this survey, which is already too long. However, I think it will be useful for the reader if we list a number of widely applicable set-theoretic or topological principles which hold under $P F A(S)[S]$ :

(1) OCA [Far96].

(2) Every Aronszajn tree is special [Far96].

(3) $\mathfrak{b}=\aleph_{2}=2^{\aleph_{0}}[$ Lar99].

(4) $P$-ideal dichotomy [Tod].

(5) Every compact, countably tight space is sequential [Tod].

(6) Every compact hereditarily separable space is hereditarily Lindelöf [Tod].

(7) Every first countable hereditarily Lindelöf space is hereditarily separable [LTo02].

(8) $\sum[$ Tod $]$ plus [Fis].

(9) Normal first countable spaces are $\aleph_{1}$-collectionwise Hausdorff [LTaa].

If $\operatorname{PFA}(S)$ is forced in the usual fashion [Dev83, Lav78], we also obtain [LTab]:

(10) Fleissner's Axiom $R$.

By starting with a particular ground model, we can obtain all the above consequences, and in addition:

(11) Every first countable normal space is collectionwise Hausdorff.

Proof [LTaa]. We first force to make a supercompact $\kappa$ indestructible under $\kappa$ closed forcing [Lav78]. We then force $G C H$ and $\diamond$ for stationary systems at all regular uncountable $\lambda$ by adjoining $\lambda^{+}$Cohen subsets of $\lambda$ for all such $\lambda$ via an Easton extension. Getting $G C H$ is standard; obtaining $\diamond$ for stationary systems is due to W. Fleissner and can be found in [Tal88]. That the supercompactness of $\kappa$ is preserved by this forcing is because it is a product of $\kappa$-closed forcing with a forcing of size $<\kappa$. ("Mild" forcings preserve large cardinals — see e.g. 10.15 of [Kan94]). Since we now have $\diamond$, we can construct a coherent Souslin tree $S$ [Lar99]. We then proceed to force $P F A(S)[S]$ in the usual manner, establishing in particular that normal first countable spaces are $\aleph_{1}$-collectionwise Hausdorff. The forcing to establish $P F A(S)[S]$ is a $\kappa$-chain condition forcing of size $\kappa$, so preserves $G C H$ as well as $\diamond$ for stationary systems for all regular $\lambda \geq \kappa$ (Fleissner, see [Tal88]). By [Fle74] (or see [Tal84]), we then have that normal first countable $\aleph_{1}$-collectionwise Hausdorff spaces are collectionwise Hausdorff. But $\kappa$ now equals $\aleph_{2}$, so we are done.

\section{Preservation under forcing}

There is a very useful technique for proving preservation of covering properties (or non-covering properties) under Cohen and random reals. This technique was introduced in [DTW90a] as part of a reflection + forcing proof of the consistency of the Normal Moore Space Conjecture, and has found several applications since: [GJT98], [Iwa07], [ST], [Kad]. It almost — but not quite — yields a ground model refinement for an open cover in a forcing extension.

Definition 3.1. An $n$-dowment is a family $\mathcal{L}$ of finite antichains of $\mathbf{F n}(\kappa, 2)$ (the forcing adding $\kappa$ Cohen reals) such that:

(1) For each maximal antichain $A \subseteq \mathbf{F n}(\kappa, 2)$, there is an $L \in \mathcal{L}$ such that $L \subseteq A$, 
(2) For every $p \in \mathbf{F n}(\kappa, 2)$ such that $|\operatorname{dom} p| \leq n$ and for every collection $L_{1}, \ldots, L_{n}$ of elements of $\mathcal{L}$, there are $q_{1} \in L_{1}, \ldots, q_{n} \in L_{n}$ and there is an $r \in \mathbf{F n}(\kappa, 2)$ such that $r \leq p$ and $r \leq q_{i}$, for every $i \leq n$.

$n$-dowments are the Cohen real analogue of the fact that for random reals, given a condition $p$ with measure $>1 \backslash\left(\frac{n-1}{n}\right)^{n}, n \in \omega$, and finite antichains, $L_{1}, \ldots, L_{n}$, each with measures summing up to at least $\frac{n-1}{n}$, there are elements $q_{i} \in L_{i}$ and a condition $r$ such that $r \leq p$ and $r \leq q_{i}$. They are named in honor of Alan Dow who discovered their existence in connection with his work on remote points in Stone-Čech remainders. A proof of their existence can be found in [DTW90a]. Since the proof itself has not had any applications in our work, we omit it. The way we use $n$-dowments is, given an open cover in a forcing extension composed of ground model open sets, we create an open cover in the ground model by taking intersections of the possible elements of the given cover given by the $L_{n}$ 's, and use what we know about open covers in the ground model to force what we want to hold in the extension. Here is a typical proof; more difficult ones can be found in the papers cited above.

Theorem 3.2. Lindelöfness is preserved by Cohen reals.

Proof. Given an open cover $\mathcal{U}$ in the extension, without loss of generality we may assume $\mathcal{U}$ is composed of ground model open sets. For each $x \in X$ we can pick a maximal antichain $A_{x}$ and open sets $U_{p, x}$ containing $x$ such that for every $p \in A_{x}$, $p \Vdash \check{U}_{p, x} \in \dot{\mathcal{U}}$. For each $n \in \omega$, let $\mathcal{L}_{n}$ be an $n$-dowment. Then for each $n \in \omega$, there is an $A_{n, x} \subseteq A_{x} . A_{n, x} \in \mathcal{L}_{n}$. Define

$$
V_{n, x}=\bigcap\left\{U_{p, x}: p \in A_{n, x}\right\} .
$$

Then $\left\{V_{n, x}: x \in X\right\}$ is an open cover of $X$ in $V$ and so has a countable subcover $\mathcal{V}_{n}$.

Claim 3.3. $\mathbb{1} \Vdash(\forall y \in X)(\exists n \in \omega)\left(\exists V \in \mathcal{V}_{n}\right)(\exists U \in \dot{\mathcal{U}})(y \in \check{V} \subseteq U)$.

Proof. We have to prove that:

$$
(\forall y \in X)(\forall q)(\exists r \leq q)(\exists n \in \omega)\left(\exists V \in \mathcal{V}_{n}\right)(\exists \text { an open } U)(r \Vdash \check{y} \in \check{V} \subseteq \check{U} \in \dot{\mathcal{U}}) .
$$

Fix $y \in X$ and $q \in \mathbf{F n}(\kappa, 2)$. Take $n \geq|d o m q|$. Since $\mathcal{V}_{n}$ covers $X$, there is a $V_{n x} \in \mathcal{V}_{n}$, such that $y \in V_{n, x}$. By the definition of $n$-dowment, there is a $p \in A_{n, x}$ such that $p$ and $q$ are compatible. Let $r \leq p, q$. Then $r \Vdash \check{y} \in \check{V}_{n, x} \subseteq \check{U}_{n, x} \in \dot{\mathcal{U}}$.

By the claim, in $V[G]$, for each $y \in X$, pick $V_{n, x}^{y}$ such that $y \in V_{n, x}^{y} \subseteq U$, for some $U \in \mathcal{U}$. Then $\left\{V_{n, x}^{y}: y \in Y\right\}$ is a countable refinement of $\mathcal{U}$, so $\mathcal{U}$ has a countable subcover.

The early work on $n$-dowments might lead one to believe that covering properties behave the same under Cohen and random extensions. This is not the case; there are several counterexamples in [ST].

Normality can be thought of as a covering property. Let's show that if $Y$ is a closed discrete subspace of $X$, and there exists a $Z \subseteq Y$ such that there do not exist disjoint open sets about $Z$ and $Y-Z$, then the same is true after adding Cohen reals.

In fact, for future use, let's prove a more complicated version of this: 
Definition 3.4. A discrete collection $\mathcal{Y}$ is normalized if for each $\mathcal{Z} \subseteq \mathcal{Y}$, there exist disjoint open sets about $\bigcup\{Y: Y \in \mathcal{Z}\}$ and $\bigcup\{Y: Y \in \mathcal{Y}-\mathcal{Z}\}$. $\mathcal{Y}$ is separated if there exist disjoint open sets $U_{Y} \supseteq Y$, for all $Y \in \mathcal{Y}$.

Theorem 3.5. If $\mathcal{Y}$ is not normalized, it remains not normalized in any Cohen real extension.

Proof. Let $\mathcal{Z} \subseteq \mathcal{Y}$ witness that $\mathcal{Y}$ is not normalized in the ground model. First consider the case of adding $\kappa$ Cohen reals where $\kappa \geq|\bigcup \mathcal{Y}|$. Let $f$ be a supposed normalization in the extension, i.e. $f: \bigcup\{Y: Y \in \mathcal{Y}\} \rightarrow \mathcal{J}$, where $\mathcal{J}$ is the ground model topology, and $\bigcup\{f(y): y \in Y \in \mathcal{Z}\} \cap \bigcup\{f(y): y \in Y \in \mathcal{Y}-\mathcal{Z}\}=0$. We can assume this, because $\mathcal{J}$ forms a basis for the topology in the extension. Using a 2-dowment $\mathcal{L}_{2}$ and considering maximal antichains $A_{y}$ of conditions deciding the value of $f$ at $y \in Y \in \mathcal{Y}$, we can define $g: \bigcup\{Y: Y \in \mathcal{Y}\} \rightarrow \mathcal{J}$ in the ground model as follows for each $y \in \bigcup\{Y: Y \in \mathcal{Y}\}, L_{y} \in \mathcal{L}_{2}, L_{y} \subseteq A_{y}$.

Let $g(y)=\bigcap\left\{U\right.$ : for some $\left.p \in L_{y}, p \Vdash \dot{f}(\check{y})=\check{U}\right\}$. We claim $\bigcup\{g(y): y \in Y \in$ $\mathcal{Y}\} \cap \bigcup\{g(y): y \in Y \in \mathcal{Y}-\mathcal{Z}\}=0$. We may assume without loss of generality (by modifying the name for $f$, if necessary) that $\mathbb{1} \Vdash \dot{f}$ is a normalization. Take $r \leq q_{0}, q_{1}$, where $q_{0} \in L_{y_{0}}$ and $q_{1} \in L_{y_{1}}$, where $y_{0} \in Y_{0} \in \mathcal{Z}$ and $y_{1} \in Y_{1} \in \mathcal{Z}-\mathcal{Y}$. Then $r \Vdash \check{g}\left(\check{y}_{0}\right) \subseteq \dot{f}\left(\check{y}_{0}\right)$ and $\check{g}\left(\check{y}_{1}\right) \subseteq \dot{f}\left(\check{y}_{1}\right)$, so $r \Vdash \check{g}\left(\check{y}_{0}\right) \cap \check{g}\left(\check{y}_{1}\right)=0$ so indeed $g\left(y_{0}\right) \cap g\left(y_{1}\right)=0$.

Now, consider the case when fewer than $\bigcup \bigcup \mathcal{Y} \mid$ reals are added. Add $|\bigcup \mathcal{Y}|$ more reals and get that $\mathcal{Y}$ is not normalized. But if there were disjoint open sets about $\bigcup \mathcal{Z}$ and $\bigcup\{\mathcal{Y}-\mathcal{Z}\}$ before adding the extra reals, these open sets would still be disjoint after the extra addition.

A similar argument proves:

Theorem 3.6. If $\mathcal{Y}$ is not separated, it remains not separated in any Cohem real extension.

Analogous results hold for random reals.

One of the early preservation results was for property $K$ forcing. A partial order $P$ satisfies property $K$ if every uncountable subset of $P$ includes an uncountable pairwise compatible uncountable subset. It is not difficult to show that both Cohen and random forcing satisfy property $K$, and it is by a now classic result that $M A_{\omega_{1}}$ implies all countable chain condition partial orders satisfy property $K$. Property $K$ forcing preserves Souslin trees, since an uncountable chain or antichain in the extension yields one in the ground model [KT79]. Since this is an exercise in [Kun80], we shall leave it to the reader. As you will see, if some ground model object has an uncountable subset in the extension satisfying some "binary property", it will also have such a subset in the ground model.

Here is the key idea in property $K$ preservation, followed by an application.

Theorem 3.7. Suppose $X \in V$ and we force with a property $K$ partial order $P$. Suppose $Y \in[X]^{\aleph_{1}}$ in the extension. Then:

$$
\left(\exists S \in[X]^{\omega_{1}}\right)\left(\forall T \in[S]^{\omega_{1}}\right)(\exists p \in P)\left(p \Vdash|T \cap Y|=\aleph_{1}\right)
$$

Proof. Let $\mathbb{1} \Vdash$ “ $\dot{f}: \check{\omega}_{1} \rightarrow \check{X}, \dot{f}$ one-one, $\dot{f} " \check{\omega}_{1}=\dot{Y}$ ". For each $\alpha \in \omega_{1}$, take a maximal incompatible family of conditions $\left\{p_{\alpha n}\right\}_{n<\omega}$ and $x_{\alpha n} \in X, n<\omega$, such that $p_{\alpha n} \Vdash \dot{f}(\check{\alpha})=\check{x}_{\alpha n}$. Let $S=\left\{x_{\alpha n}: \alpha<\omega_{1}, n<\omega\right\}$. Then $S$ is uncountable, 
for take an uncountable pairwise compatible subset of $\left\{p_{\alpha n}: \alpha<\omega_{1}, n<\omega\right\}$ and observe that the corresponding $x_{\alpha n}$ 's are distinct. Now suppose, on the contrary, that for some $T \in[S]^{\omega_{1}}, \mathbb{1} \Vdash \dot{f}$ '“ $\breve{\omega}_{1} \cap \check{T}$ is countable. Extend each $p_{\alpha n}$ such that $x_{\alpha n} \in T$ to a $q_{\alpha n}$ for which there is a $\beta_{\alpha n}$ such that for each $\gamma \geq \beta_{\alpha n}$, $q_{\alpha n} \Vdash \dot{f}(\check{\gamma}) \notin \check{T}$. Since $T$ is uncountable, there is an uncountable $A \subseteq \omega_{1} \times \omega$ such that $\left\{q_{\alpha n}:\langle\alpha, n\rangle \in A\right\}$ is pairwise compatible. But now take some $\left\langle\alpha_{0}, n_{0}\right\rangle \in A$, and then take $\left\langle\alpha_{1}, n_{1}\right\rangle \in A$ such that $\alpha_{1} \geq \beta_{\alpha_{0} n_{0}}$. Then $x_{\alpha_{1} n_{1}} \in T$ and $q_{\alpha_{1} n_{1}} \Vdash$ $\dot{f}\left(\check{\alpha}_{1}\right)=\check{x}_{\alpha_{1} n_{1}}$, but $q_{\alpha_{0} n_{0}} \Vdash \dot{f}\left(\check{\alpha}_{1}\right) \notin T$, contradiction.

Corollary 3.8 ([BT02]). Suppose $X$ is a space in $V$ and we force with a property $K$ partial order. Then if $X$ has a Lindelöf subspace of size $\aleph_{1}$ in the extension, it has one in $V$ as well.

Proof. Suppose $Y \in[X]^{\aleph_{1}}$ is Lindelöf in the extension. Form $S$ as above. Claim $S$ is Lindelöf. We know that every uncountable $T \subseteq S$ is forced to have uncountable intersection with $f$ " $\omega_{1}$ in some extension. Then, in that extension, $T$ is forced to have a complete accumulation point in $f$ " $\omega_{1}$. Thus there are $p, x, \alpha$ in $V$ such that $p \Vdash \dot{f}(\check{\alpha})=\check{x}$ and $\check{x}$ is a complete accumulation point of $\check{T}$. But then $x$ really is a complete accumulation point of $T$, and since $p$ is compatible with some $p_{\alpha n}$, $x=x_{\alpha n}$ is in $S$. Thus $S$ is a subspace of size $\aleph_{1}$ in which every uncountable set has a complete accumulation point, so $S$ is Lindelöf.

Another strengthening of the countable chain condition which is of interest from the point of view of preservation is that a partial order $P$ be $\sigma$-centered. i.e. $P=\bigcup\left\{P_{n}: n<\omega\right\}$, where for each $n$ and each finite $F \subseteq P_{n}, F$ has a lower bound in $P$ (not necessarily in $P_{n}$ ). Again it is a classic result that $M A_{\omega_{1}}$ implies every subset of size $\aleph_{1}$ of a countable chain condition partial order is $\sigma$-centered. This weakening of " $\sigma$-centered" is preserved by arbitrary finite-support products, but $\sigma$-centered itself is not. These can be seen by looking at the analogous results for separable topological spaces.

It is quite clear how to try to show that a covering property in a $\sigma$-centered extension pulls down to the ground model: it gives rise to countably many collections of open sets in the ground model, each having finitary properties like the original object in the extension. Suppose for example that in the extension, every open cover of a ground model space $X$ has a $\sigma$-disjoint open refinement. We claim the same is true in the ground model. Let $\mathcal{U}$ be an open cover of $X$ in $V$. Without loss of generality, we may assume its $\sigma$-disjoint refinement $\left\{\mathcal{U}_{n}: n<\omega\right\}$ in the extension is composed of ground model open sets. Let $P=\bigcup_{n<\omega} P_{n}$, where each $P_{n}$ is centered. Let $\mathcal{W}_{n m}=\left\{W: W\right.$ is open in $V$ and for some $\left.p \in P_{m}, p \Vdash \check{W} \in \dot{\mathcal{U}}_{n}\right\}$.

Claim 3.9. $\mathcal{W}=\bigcup\left\{\mathcal{W}_{n m}: n, m<\omega\right\}$ is a $\sigma$-disjoint open refinement of $\mathcal{U}$.

Proof. First of all, $\mathcal{W}$ is a refinement, since $p \Vdash \check{W} \in \dot{\mathcal{U}}_{n}$ implies $p \Vdash(\exists U \in \check{\mathcal{U}})(\check{W} \subseteq$ $U)$, so

But then $W \subseteq U \in \mathcal{U}$.

$$
(\forall q \leq p)(\exists r \leq q)(\exists U \in \mathcal{U}) r \Vdash \check{W} \subseteq \check{U}
$$

Second, $\mathcal{W}$ is an open cover, since $\mathbb{1} \Vdash \bigcup\left\{\dot{\mathcal{U}}_{n}: n<\omega\right\}$ is a cover, so $\mathbb{1} \Vdash(\forall x \in$ $\check{X})(\exists n \in \omega)\left(\exists U \in \dot{\mathcal{U}}_{n}\right)(x \in \check{U})$. Then $(\forall x \in X)(\forall p)(\exists q \leq p)(\exists n)(\exists U)\left(q \Vdash \check{U} \in \dot{\mathcal{U}}_{n}\right.$ and $\check{x} \in \check{U})$. Then $x \in U$, and for some $m, q \in P_{m}$, so $x \in U \in \mathcal{W}_{n m}$.

Finally, each $\mathcal{W}_{n m}$ is disjoint, for if $p_{1} \Vdash \check{W}_{1} \in \dot{\mathcal{U}}_{n}$ and $p_{2} \Vdash \breve{W}_{2} \in \dot{\mathcal{U}}_{n}$, where $p_{1}, p_{2} \in P_{m}$, take $q \leq p_{1}, p_{2}$. Then $q \Vdash \check{W}_{1} \cap \check{W}_{2}=0$, so indeed, $W_{1} \cap W_{2}=0$. 


\section{SuperCompaCt REFLECTION}

Quite often one would like to prove the consistency of some universal statement, e.g. "every hexacompact space with a purple diagonal (an HP space) is foreclosable". The first step would be to consider a particular hexacompact space with a purple diagonal and force it to be foreclosable. Another possibility would be to start with a space which is not foreclosable, and force it to either be non-hexacompact or to not have a purple diagonal. Assume you could, for example, do the foreclosable forcing. If your forcing were sufficiently nice, you would be able to iterate it to force other hexacompact spaces with purple diagonals to be foreclosable, but there are some obvious difficulties in the way of establishing your desired consistency result.

(1) There could be too many $H P$ spaces, so you could never deal with them all.

(2) The very act of making one $H P$ space foreclosable could create other $H P$ spaces, so that you are facing an unwinnable game of Whack-a-mole.

(3) Once you "kill" an $H P$ space by making it foreclosable, perhaps it doesn't stay "dead", but rather is "revived", i.e. made non-foreclosable again, by later forcing.

Each of these problems needs to be dealt with; in some - but not all - circumstances, this can be accomplished. If the number of $H P$ spaces is bounded, \#1 is doable. For example, if every $H P$ space has size $\leq \aleph_{1}$, and $2^{\aleph_{1}}=\aleph_{2}$, once could deal with them all in an iteration of length $\aleph_{2}$. One could also do a bookkeeping argument as in the consistency proof for $M A_{\omega_{1}}$ to take care of $\# 2$.

If the number of $H P$ spaces is unbounded, there are two reasonable approaches. One is to do class forcing: deal with all HP spaces of size $\aleph_{1}$ in $\aleph_{2}$ steps, all those of size $\aleph_{2}$ in $\aleph_{3}$ steps, etc. For an example of this sort of argument, see [Tal88]. A more widely applicable technique is to use reflection: show that there is a cardinal $\kappa$ such that if every $H P$ space of size $<\kappa$ is foreclosable, then every $H P$ space is. One could then plausibly get that every $H P$ space is foreclosable by an iteration of length $\kappa$. One might be able to achieve such reflection by an elementary submodel argument, but, in general, one is likely to have to assume the existence of a large cardinal, in particular a supercompact one, and so we turn our attention to that.

Since the existence of large cardinals entails the existence of models of $Z F C$, by Gödel's Incompleteness Theorem, such existence cannot be proved consistent. Thus, proponents of large cardinal axioms must convince other mathematicians that they should adopt such axioms, or at least that their adoption is harmless. The arguments of the proponents resemble those of medieval philosophers for the existence of God; rather than explore that parallel, we shall follow the contemporary set-theoretic fashion and sleep soundly despite using supercompact cardinals. However, it seems interesting to note that the concept of reflection also appears in the Kabbalah: as above, so below, i.e. what happens in Heaven is reflected here on Earth.

A typical use of supercompact cardinals in topology is to prove results of the form if every small subspace of $X$ has property $P$, then $X$ has property $P$. "Small" here means of size $<\kappa$, where $\kappa$ is the supercompact cardinal. Such results are called compactness theorems. Taking the contrapositive, we obtain if $X$ has property $Q$ $(=\sim P)$, then some small subspace of $X$ has the property $Q$. This is called a reflection theorem. Such results are not so interesting, since $\kappa$ is very large. The 
game, however, is to make $\kappa$ relatively small, either by collapsing it to $\aleph_{1}$ or $\aleph_{2}$, or by blowing up the continuum to have size $\kappa$, and hope that the compactness/reflection results for that particular $P$ survive the collapse or blow-up. Such results have a pattern worth learning, which we shall now explain. The pattern involves general machinery concerning elementary embeddings.

Definition 4.1. An injection $j$ from a model $V$ into a model $M$ of the same language is an elementary embedding if for each $n$, for every formula $\phi$ with $n$ free variables, and any $n$ elements $x_{1}, \ldots, x_{n} \in V$,

$$
V \vDash \phi\left[x_{1}, \ldots, x_{n}\right] \text { if and only if } M \vDash \phi\left[j\left(x_{1}\right), \ldots, j\left(x_{n}\right)\right] .
$$

Definition 4.2. A cardinal $\kappa$ is supercompact if there is for each cardinal $\lambda \geq \kappa$ an elementary embedding $j_{\lambda}: V \rightarrow M$ from the universe $V$ into a transitive class $M$ such that:

- for each $\xi<\kappa, j_{\lambda}(\xi)=\xi$, but $j_{\lambda}(\kappa)>\lambda$, and

- ${ }^{\lambda} M \subseteq M$.

Here, ${ }^{\lambda} M$ denotes the class of sequences of length $\lambda$, where the terms of the sequences are elements of $M$. It follows by transfinite induction that for all $\alpha \leq \lambda$ we have $M_{\alpha}=V_{\alpha}$ - that is, the cumulative hierarchy as computed in $V$ and in $M$ coincides at least up to $\lambda$.

The above definition of supercompactness is the most useful for applications, but prima facie is not expressible in $Z F C$ because of the quantification over formulas. There is an equivalent definition which asserts the existence of certain ultrafilters and thus avoids this difficulty. See [Kan94].

A typical proof using supercompactness exploits the interplay between $j$ and $j$ ", where:

$$
j^{*} X=\{j(x): x \in X\} .
$$

Clearly $j(X) \supseteq j$ " $(X)$, since if $x \in X$, by elementarity, $j(x) \in j(X)$. Also, $j$ " $\kappa=\kappa$ but $j(\kappa)>\kappa$ if $\kappa$ is supercompact and $j$ is one of the $j_{\lambda}$ 's, $\lambda \geq \kappa$. Thus $j$ " $X$ is a copy of $X$, while $j(X)$ "looks just like $X$ " (by elementarity) but can be much bigger, if $|X| \geq \kappa$. We then have that $j(X)$ has a smaller subset, namely $j$ " $X$, that looks like it, so by elementarity, $X$ must have such a subset as well. If $\phi$ is a first-order property of $X$, both $j(X)$ and $j$ " $X$ will satisfy $\phi$, so we will be able to conclude that $j(X)$ - and hence $X$ - has a subset of smaller size satisfying $\phi$.

The following are frequently used facts about elementary embeddings. We leave the proofs to the reader as an exercise, but they can be found in [DTW90a].

Lemma 4.3. Suppose $j: V \rightarrow M$ is an elementary embedding into a transitive class $M$. Then:

(i) For any $A \in V, j$ " $A \subseteq j(A)$,

(ii) $A \subseteq B \in V \rightarrow j(A) \cap j$ " $B=j$ " $A$

(iii) if $j(\alpha)=\alpha$ for all $\alpha<\kappa$ and $A \in V$ is such that $|A|<\kappa$, then $j(A)=j$ " $A$.

(iv) if $j(\alpha)=\alpha$ for all $\alpha<\kappa$, then for any $a \in V$ of rank $<\kappa, j(a)=a$.

After we force with our partial order $\mathbb{P}$ to collapse $\kappa, \kappa$ is no longer supercompact in the extension. We would like to make use of its former supercompactness. To do this, we seek to extend the elementary embedding $j: V \rightarrow M$ to $V[G]$, where $G$ is $\mathbb{P}$-generic over $V$.

We cannot expect to extend $j$ to an embedding $j^{*}: V[G] \rightarrow M[G]$, since $M[G]$ is a transitive subclass of $V[G]$ and so such a $j^{*}$ would demonstrate that $\kappa$ is still 
supercompact in $V[G]$. Instead we will (when certain conditions are met) extend $j$ to $j^{*}: V[G] \rightarrow M\left[G^{*}\right]$, where $M\left[G^{*}\right]$ is a generic extension of $M[G]$ (for instance, if $G$ adds $\kappa$ Cohen reals, then $G^{*}$ adds $j(\kappa)$ Cohen reals). The details and conditions are developed below.

Definition 4.4. $j^{*}$ is a generic elementary embedding if there exists some $H$ which is generic over $M[G]$ such that $j^{*}: V[G] \rightarrow M[G][H]$.

The following technical lemmas give us sufficient conditions for extending an elementary embedding to a generic elementary embedding.

Lemma 4.5. Let $j$ be an elementary embedding from $V$ into a transitive class $M$. Suppose $G$ is $\mathbb{P}$-generic over $V$ and $G^{*}$ is $j(\mathbb{P})$-generic over $M$. If $p \in G \rightarrow j(p) \in$ $G^{*}$ for all $p \in \mathbb{P}$, then $j$ extends to an elementary embedding $\tilde{\jmath}: V[G] \rightarrow M\left[G^{*}\right]$.

Proof. If $x=\operatorname{val}(\tau, G)$, define $\tilde{\jmath}(x)=\operatorname{val}\left(j(\tau), G^{*}\right)$.

Lemma 4.6. Let $j: V \rightarrow M$ be an elementary embedding with critical point (the first ordinal moved) $\kappa$. Let $G$ be $\mathbb{P}$-generic over $V$. If $j(\mathbb{P})$ is equivalent to (i.e., yields the same extension as) $j$ " $\mathbb{P} * \mathbb{Q}$ for some $\mathbb{Q}($ call it $j(\mathbb{P}) / j$ $\mathbb{P}$ ) and there is a master condition $m \in \mathbb{Q}$ such that if $H$ is a $\mathbb{Q}$-generic filter over $M[G]$ containing $m$, then $p \in G \rightarrow j(p) \in G * H$, then $G * H$ is $j(\mathbb{P})$-generic over $M$.

In particular, this is true if $\mathbb{P}$ is $\kappa$-c.c.

For the $\kappa$-c.c. case, the element $\mathbb{1} \in \mathbb{Q}$ is a master condition.

Often, the master condition will look like $\bigcup G$, where $G$ is $\mathbb{P}$-generic over $V$, although sometimes extra work is necessary in order for this to make sense. For example, in the case that $\mathbb{P}$ is the usual poset to collapse $\kappa$ to $\omega_{2}, \bigcup G$ is not a condition in the second poset. Silver solved that problem by modifying the Lévy collapse to the Silver collapse. For this and much more that was supposed to be in the second volume of Kanamori [Kan94], see [KM78].

Let's verify this in the simple case when $\mathbb{P}$ is $\mathbf{F n}(\kappa, 2)$, the partial order for adjoining $\kappa$ Cohen reals. Suppose $H$ is any $\mathbb{Q}$-generic filter over $M[G]$ where $G$ is $\mathbb{P}$-generic over $V$. If $p \in G$, then $j(p)=p$, so $j$ " $\mathbb{P}=\mathbb{P}$ and $j(\mathbb{P})=\mathbf{F n}(j(\kappa), 2)=$ $\boldsymbol{F n}(\kappa, 2) * \mathbf{F n}(j(\kappa) \backslash \kappa, 2)$. If $H$ is any $\mathbf{F n}(j(\kappa) \backslash \kappa, 2)$-generic over $M[G]$ set (all such sets contain $\mathbb{1}$, the empty condition), then $p \in G$ implies $j(p)(=p) * \mathbb{1} \in G * H$.

Having developed the general machinery, let's start reaping the rewards.

Let us first examine this interplay in the simpler context in which we don't do any forcing. Then, given a topological space $\langle X, \mathcal{J}\rangle, j$ " $\mathcal{J}$ is a topology on $j$ " $X$, and $\langle j " X, j " \mathcal{J}\rangle$ is homeomorphic to $\langle X, \mathcal{J}\rangle .\langle j(X), j(\mathcal{J})\rangle$ is also a topological space; if $\langle j$ " $X, j$ " $\mathcal{J}\rangle$ were a subspace of $\langle j(X), j(J)\rangle$, we could argue that since $\langle j$ " $X, j$ " $\mathcal{J}\rangle$ is homeomorphic to $\langle X, \mathcal{J}\rangle$, if $\langle X, \mathcal{J}\rangle$ has property $P$, so does $\langle j " X, j$ “ $\mathcal{J}\rangle$. On the other hand, by elementarity, if $\langle X, \mathcal{J}\rangle$ has property $P$, so does $\langle j(X), j(\mathcal{J})\rangle$. Thus, taking $j$ such that $j(\kappa)>|X \cup \mathcal{J}|$, we could get that $\langle j(X), j(\mathcal{J})\rangle$ would have a subspace of size $<j(\kappa)$ with property $P$, whence by elementarity $\langle X, \mathcal{J}\rangle$ would have a subspace of size $<\kappa$ with property $P$.

The question, then, is when is $j$ " $X$ a subspace of $j(X)$ ? (We shall omit mentioning the topology when it is clear from context.) There is a useful sufficient condition:

Theorem 4.7. If each point of $X$ has character less than $\kappa$, the critical point of the elementary embedding $j$, then $j$ " $X$ is a subspace of $j(X)$. 
Proof. Let $\{N(p, \alpha)\}_{\alpha<\mu}, \mu<\kappa$, be a neighbourhood base at $p \in X$. Then $j(N(p, \alpha))_{\alpha<j(\mu)}$ is a neighbourhood base at $j(p)$ in $j(X) .\left\{j^{\prime \prime}(N(p, \alpha))\right\}_{\alpha<\mu}$ is a neighbourhood base at $j(p)$ in $j$ " $X . j(\mu)=\mu$ and $j(N(p, \alpha)) \cap j$ " $X=j$ " $(N(p, \alpha))$.

We shall illustrate the general procedure with a result from a recent paper [ST]. A classic problem of Hajnal and Juhász [HJ76] asks whether every Lindelöf space has a Lindelöf subspace of size $\aleph_{1}$. There are some positive results for special cases or with additional set-theoretic hypotheses in [BT02], but whether there is a consistent positive answer in general is unknown. A consistent counterexample was constructed in [KT02]. We shall strengthen "Lindelöf" to "Rothberger" and show that the general machinery establishes:

Theorem 4.8. If it's consistent that there is a supercompact cardinal, it's consistent that every Rothberger space of character $\leq \aleph_{1}$ has a Rothberger subspace of size $\aleph_{1}$.

Definition 4.9. A space is Rothberger if whenever $\left\{\mathcal{U}_{n}: n<\omega\right\}$ is a sequence of open covers, there are $U_{n} \in \mathcal{U}_{n}, n<\omega$ such that $\left\{U_{n}: n<\omega\right\}$ is a cover.

The Rothberger property was introduced in the context of the real line by Fritz Rothberger; it is studied in detail in [ST], in which further references can be found.

Clearly, Rothberger spaces are Lindelöf, but more is true:

Lemma 4.10. After countably closed forcing, a Rothberger space $X$ remains Rothberger.

Given $\left\{\mathcal{U}_{n}\right\}_{n<\omega}$ in the extension, we may assume each $\mathcal{U}_{n}$ is composed of ground model open sets. If $X$ is still Lindelöf, we will be able to find countable subcovers for each $\mathcal{U}_{n}$, and then go back and apply Rothberger in the ground model. Thus we need only to show that the Lindelöf property of Rothberger spaces is preserved by countably closed forcing. A good way to see this is via certain topological games. We follow the treatment in [ST]. Consider an $\alpha$-inning game, $\alpha$ an ordinal. Player I picks an open cover, Player II picks a member of that cover, etc., for each $\beta<\alpha$. Player II wins if her choices form an open cover; Player I wins otherwise.

Lemma 4.11 ([Paw94]). A space is Rothberger if and only if Player I has no winning strategy in the $\omega$-length version of the covering game.

More surprising is:

Theorem 4.12 ([ST]). A space $X$ is Lindelöf in all countably closed forcing extensions if and only if Player I has no winning strategy in the $\omega_{1}$-length covering game for $X$.

If Player I has no winning strategy in the $\omega_{1}$-length game, she has no winning strategy in the $\omega$-length game, so by the above remarks, we see that:

Corollary 4.13. The Rothberger property is preserved by countably closed forcing.

Theorem 4.12 is proved from a result in [Tal95], which in turn is based on [She96]. We need the following definition:

Definition 4.14. A collection $\left\{U_{f}: f \in \bigcup_{\alpha<\omega_{1}}{ }^{\alpha} \omega\right\}$ of open subsets of $X$ is a covering tree if for every $\alpha \in \omega_{1}$, and every $f \in{ }^{\alpha} \omega,\left\{U_{f}\{\langle\alpha, n\rangle\}: n<\omega\right\}$ is a cover of $X$. 
Lemma 4.15 ([Tal95]). Let $X$ be a Lindelöf space. The following are equivalent:

(1) $X$ is Lindelöf in all countably closed forcing extensions,

(2) $X$ is Lindelöf after adding one Cohen subset of $\omega_{1}$,

(3) for each covering tree $\left\{U_{f}: f \in \bigcup_{\alpha<\omega_{1}}{ }^{\alpha} \omega\right\}$ of $X$, there is an $f \in \bigcup_{\alpha<\omega_{1}}{ }^{\alpha} \omega$ such that $\left\{U_{f \mid \beta}: \beta \in \operatorname{dom} f\right\}$ covers $X$.

Proof. Trivially, a) implies b). Let's show not a) implies not c). Suppose that in some countably closed forcing extension, there is an open cover $\mathcal{U}$ which has no countable subcover. We may assume without loss of generality that it is composed of ground model open sets. Form a tree indexed by $\bigcup_{\beta<\omega_{1}}{ }^{\beta} \omega$, with each node assigned a member $p_{f}$ of the offending poset as follows. At each node $f$ pick countably many conditions extending $p_{f}$ and deciding $p_{f}$ and deciding some element of $\mathcal{U}$. By Lindelöfness, we can pick those conditions such that the decided elements cover. At limit levels, use countable closure to pick a condition below the conditions on a branch. The resulting covering tree witnesses the failure of $\mathrm{c}$ ).

To see that not c) implies not b), note that a Cohen subset of $\omega_{1}$ determines a branch through the tree. By genericity, the open sets decided by that branch form a cover which has no countable subcover, else some $p_{f}$ would force that $\mathcal{U}$ had a countable subcover. We have thus shown a) $\Rightarrow b) \Rightarrow c) \Rightarrow a$ ).

From Lemma 4.15, we can easily prove the important direction of Theorem 4.12 , for let $\left\{U_{f}: f \in \bigcup_{\alpha<\omega_{1}}{ }^{\alpha} \omega\right\}$ be a covering tree of $X$. This implicitly defines a strategy for Player I in the $\omega_{1}$-length covering game; a winning play against this strategy yields a cover, but then Lindelöfness cuts it down to some $\alpha$-length branch. We omit the other direction.

For $\lambda<\kappa$ infinite regular cardinals let $\mathbf{L v}(\kappa, \lambda)$ be the partially ordered set whose elements are functions $p$ such that $|p|<\lambda$, $\operatorname{dom}(p) \subseteq \kappa \times \lambda$ and for all $(\alpha, \xi) \in \operatorname{dom}(p), p(\alpha, \xi) \in \alpha$, elements $p$ and $q$ are ordered by $p \leq q$ if and only if $q \subseteq p$. It is well-known that when $\kappa$ is strongly inaccessible, each antichain of $\mathbf{L v}(\kappa, \lambda)$ is of cardinality less than $\kappa$. Also, as $\lambda$ is regular, $\mathbf{L v}(\kappa, \lambda)$ is $\lambda$-closed. It is also well-known that in the generic extension obtained by forcing with $\mathbf{L v}(\kappa, \lambda)$, $\kappa$ remains a cardinal, but is the successor of $\lambda$. The phrase "Lévy-collapse ... to $\omega_{2}$ with countable conditions" means "Force with $\mathbf{L v}\left(\kappa, \omega_{1}\right)$ where $\kappa$ is supercompact".

Theorem 4.16. Lévy-collapse a supercompact cardinal to $\omega_{2}$ with countable conditions. Then every Rothberger space of character $\leq \aleph_{1}$ includes a Rothberger subspace of size $\leq \aleph_{1}$.

Proof. Let $\kappa$ be a supercompact cardinal in the ground model. Let $G$ be $\mathbf{L v}\left(\kappa, \omega_{1}\right)$ generic over $V$. In $V[G]$, let $\langle X, \mathcal{J}\rangle$ be a Rothberger space of character $\leq \aleph_{1}$ and cardinality larger than $\aleph_{1}$. Let $\alpha$ be a cardinal such that $X$, all open covers of $X$, and all sequences of open covers of $X$, as well as $\mathbf{L v}\left(\kappa, \omega_{1}\right)$ and any of its antichains are members of $V_{\alpha}[G]$. Choose a regular cardinal $\lambda>2^{\alpha}$. In $V[G]$ let $\mu=|X|$. Then $\mu$ is also a cardinal in $V$ and in $V, \kappa \leq \mu<\alpha<\lambda$.

By supercompactness of $\kappa$, fix an elementary embedding $j: V \rightarrow M$ with $j(\kappa)>$ $\lambda$ and ${ }^{\lambda} M \subseteq M$. Since for each $\beta \leq \lambda$ we have $M_{\beta}=V_{\beta}$, it follows that $\mathbf{L v}\left(\kappa, \omega_{1}\right)$ is $\mathbf{L v}\left(\kappa, \omega_{1}\right)$-generic over $M$ if and only if it is over $V$. Moreover, for all $\beta \leq \lambda$, $V_{\beta}[G]=M_{\beta}[G]$. It follows that in fact $X \in M[G]$. Moreover, any family of open covers for $X$ in $V[G]$ is also in $M[G]$. Thus, $M[G] \vDash$ " $X$ is a Rothberger space of cardinality $>\aleph_{1}$ and character $\leq \aleph_{1}{ }^{\prime}$. 
Since $\mathbf{L v}\left(\kappa, \omega_{1}\right)$ has no antichain of cardinality $\kappa$, there is a $j\left(\mathbf{L v}\left(\kappa, \omega_{1}\right)\right)$-generic (over $M$ ) filter $G^{*}$ such that $p \in G$ implies $j(p) \in G^{*}$. But then $j$ extends to an elementary embedding we shall also call $j: V[G] \rightarrow M\left[G^{*}\right]$.

Until further notice we now work in $M\left[G^{*}\right]$. The equation $j\left(\operatorname{Lv}\left(\kappa, \omega_{1}\right)\right)=$ $\mathbf{L v}\left(j(\kappa), \omega_{1}\right)=\mathbf{L v}\left(\kappa, \omega_{1}\right) \times \mathbf{L v}\left(j(\kappa) \backslash \kappa, \omega_{1}\right)$ implies that $M\left[G^{*}\right]$ is of the form $M[G][H]$ where $H$ is $\mathbf{L v}\left(j(\kappa) \backslash \kappa, \omega_{1}\right)$-generic over $M[G]$. By Corollary 4.13, Rothberger is preserved by countably closed forcing, giving $M\left[G^{*}\right] \vDash$ " $X$ is a Rothberger space".

The bijection $\left.j\right|_{X}$ from $X$ to $j$ " $X$ induces a homeomorphic topology, say $j$ " $\mathcal{T}$, on $j$ " $X$ : Then $\left(\left.j\right|_{X}\right.$ " $\left.X, \mathcal{T}\right)$ is a Rothberger space.

The subset $j$ " $X$ of $j(X)$ inherits a topology from $j(X)$, say $\mathcal{S}$. Compare the two spaces $(j$ " $X, j$ " $\mathcal{T})$ and $(j " X, \mathcal{S})$. First note that $\mathcal{S}$ contains sets of the form $j(U) \cap j$ " $X$, where $U \subseteq X$ is open in $X$. Since $j(U) \cap j$ " $X=j$ " $U$ is an element of the topology $j$ " $\mathcal{T}$ on $j$ " $X$, we have $j$ " $\mathcal{T} \subseteq \mathcal{S}$. The character restriction ensures that $\mathcal{S}=j$ "T $\mathcal{T}$. Thus, $j$ " $X$ is a Rothberger subspace of $j(X)$.

Since $j$ is an elementary embedding, $j(X)$ is a Rothberger space with character $\leq \aleph_{1}$ and cardinality $j(\mu)$. Since $j[X]$ is an uncountable subset of $j(X)$, we conclude that in $M\left[G^{*}\right]$ the statement

" $j$ " $X \subseteq j(X)$ is an uncountable Rothberger subspace of $j(X)$ "

as well as the statement " $j(\kappa)=\aleph_{2}$ and $|\mu|=\aleph_{1}$ " are true. This implies:

- $M\left[G^{*}\right] \Vdash$ " $j(X)$ has a Rothberger subspace of cardinalty $\aleph_{1}$ ".

This concludes working in $M\left[G^{*}\right]$. Since $j\left(\aleph_{1}\right)=\aleph_{1}$ and $j$ is an elementary embedding of $V[G]$ into $M\left[G^{*}\right]$, in $V[G]$ it is true that $X$ has a Rothberger subspace of cardinality $\aleph_{1}$.

By different methods, Marion Scheepers [Sch] has recently been able to obtain this result by Lévy-collapsing only a measurable to $\aleph_{2}$.

In the Cohen or random situation, our preservation results work no matter how many reals we are adjoining. The situation is considerably more subtle in the $\sigma$-centered case, because the iteration of $\sigma$-centered partial orders need not be $\sigma$-centered. A typical example of when one would want preservation by arbitrary iterations of $\sigma$-centered partial orders is when one wants to have reflection phenomena co-existing with $M A$ ( $\sigma$-centered), or equivalently, $\mathfrak{p}=\mathfrak{c}$ [Bel81], [Wei84]. The point is that $j(\mathbb{P}) / \mathbb{P}$ - where $\mathbb{P}$ is the natural partial order that forces $\mathfrak{p}=\kappa$, where $\kappa$ is supercompact, will not be $\sigma$-centered, although it is an iteration of $\sigma$-centered partial orders, since it is an iteration of more that $2^{\aleph_{0}}$ such orders. Nonetheless, in many cases in which one has preservation by $\sigma$-centered forcing, one can actually prove preservation by finite support iterations of $\sigma$-centered forcing.

Definition 4.17. An $\alpha$-stage finite support iteration $\mathbb{P}$ with typical successor stage $\mathbb{P}_{\beta+1}=\mathbb{P}_{\beta} * \dot{\mathbb{Q}}_{\beta}$ is an absolute $\sigma$-centered iteration if in any forcing extension, for any $\beta<\alpha, \Vdash_{\beta} \dot{\mathbb{Q}}_{\beta}$ is $\sigma$-centered.

Lemma 4.18 ([Tal94a]). Suppose $\Phi(X)$ is preserved by $\sigma$-centered forcing. Let $\kappa$ be a cardinal. Suppose there is a poset $\mathbb{R}$ such that $\mathbb{R} \Vdash \kappa \leq 2^{\aleph_{0}}$ and $\mathbb{R}$ preserves both $\Phi(X)$ and $\neg \Phi(X)$. Then $\Phi(X)$ is preserved by finite support $\kappa$-stage absolute $\sigma$-centered iterations.

Proof. Let $\mathbb{P}$ be a finite support $\kappa$-stage absolute $\sigma$-centered iteration, and suppose for a contradiction that $\mathbb{P}$ does not preserve $\Phi(X)$. Then neither does $\mathbb{R} \times \mathbb{P}$; hence, 
the same goes for $\mathbb{P} \times \mathbb{R}$. But $\mathbb{R} \Vdash \kappa \leq 2^{\aleph_{0}}$, and $\mathbb{P}$ is still a finite support $\kappa$-stage $\sigma$-centered iteration after forcing with $\mathbb{R}$. Therefore after forcing with $\mathbb{R}, \mathbb{P}$ is $\sigma$ centered. This is a contradiction, since $\Phi(X)$ is preserved by $\mathbb{R}$ and by $\sigma$-centered forcing.

Thus, for example, if we can show that some forcing that adds a lot of reals preserves both $\Phi$ and $\neg \Phi(X)$, and $\sigma$-centered forcing preserves $\Phi(X)$, then arbitrary finite support iterations of $\sigma$-centered forcing preserve $\Phi(X)$. There is an application of this in [Tal94a], involving submetrizability and random reals. The random real preservation argument is novel and ought to have additional applications. Given a function $d(x, y)$ bounded by 1 in a random real extension - e.g. a metric — we define $\rho(x, y)$ in $V$ which behaves like $d$ :

$$
\rho(x, y):=\sum_{n \in N} \frac{\mu\left(\left\|\dot{d}(x, y)>\frac{1}{n}\right\|\right)}{2^{n}} .
$$

Preservation arguments akin to $\sigma$-centered ones arise in some applications of huge cardinal collapse. One can collapse $\kappa$ to $\aleph_{1}$ and $j(\kappa)$ to $\aleph_{2}$ such that $j(\mathbb{P}) / j$ " $\mathbb{P}$ is $\aleph_{1}$-centered $* \aleph_{2}$-closed. This enables the transfer of some properties - e.g. weak collectionwise Hausdorffness, from $\aleph_{1}$ to $\aleph_{2}$ [Tal94b].

\section{NORMALITY VERSUS COLLECTIONWISE NORMALITY}

Once one has "reflection + preservation" in one's mind, as well as "small character implies $j$ " $X$ is a subspace of $j(X)$ ", it is fairly easy to come up with a proof of the following result, the conclusion of which implies the Normal Moore Space Conjecture:

Theorem 5.1 ([DTW90a]). Adjoin either supercompact many Cohen reals or supercompact many random reals. Then every normal space in which each point has character $<2^{\aleph_{0}}$ is collectionwise normal.

Proof. First check that these partial orders are candidates for the method by checking that $P_{\kappa}$ is completely embedded in $j\left(P_{\kappa}\right)=P_{j(\kappa)}$. Suppose $X$ were a counterexample. Then there would be a discrete unseparated subcollection $\mathcal{Y}$ in $X$. By Theorem 3.6, "unseparated" is preserved by Cohen or random forcing. Thus $\mathcal{Z}=\{j " Y: Y \in \mathcal{Y}\}$ is unseparated in $j$ " $X$ and hence in $j(X)$. It is also easy

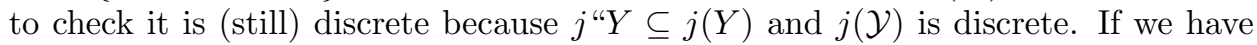
picked a $j$ that sends $\kappa$ past $|\bigcup \mathcal{Z}|$, we have $\mathcal{Z}$ is "a small discrete collection of small sets". Thus it suffices to show that if we adjoin either $\mu$ Cohen reals or $\mu$ random reals, where $\mu$ is regular, then in a normal space in which points have character $<2^{\aleph_{0}}=\mu$, discrete collections $\mathcal{Y}$ such that $|\bigcup \mathcal{Y}|<\mu$ are separated. This is established by a slight variation of the proof of Theorem 3.5 - we need to show that not only does normalized in the extension imply normalized in the ground model, but that it implies separated as well.

We will only do the Cohen argument. We may assume without loss of generality that $\mathcal{Y} \in V$. We slightly vary the proof of Theorem 3.5 to show that if $\mathcal{Y}$ is normalized in a Cohen extension, then it is separated in $V$. We may assume we have added more than $\bigcup \cup \mathcal{Y} \mid$ Cohen reals. By some easy topology, if $\mathcal{Y}=\left\{Y_{\gamma}\right\}_{\gamma<\lambda}, \lambda<\kappa$, it suffices to separate $\left\{Y_{\gamma}: \gamma \in \kappa \backslash\right.$ dom $\left.p\right\}$, where $p \Vdash \check{\mathcal{Y}}$ is normalized. Proceed as before, but also insist $r$ is below $\left\{\langle\gamma, 0\rangle,\left\langle\gamma^{\prime}, 1\right\rangle\right\}$, in order to ensure that the open sets about $Y_{\gamma}$ and $Y_{\gamma^{\prime}}, \gamma \neq \gamma^{\prime}$, are disjoint. 
Many of the interesting questions involving reflection fit into the general framework we have outlined, but others do not, because the character requirement is too restrictive. A case in point is the consistency from a supercompact cardinal of every locally compact normal space being collectionwise normal. The first proof by Balogh [Bal91] was unnecessarily difficult, and it was not clear what was going on, or whether there was any generally applicable method. The proof of Dow [Dow92] was an improvement but still was not transparent, at least not to those not embedded in $C^{*}(X)$. The proof in [GJT98] has the advantage that conceptually it is a minor modification of the small character case, namely replacing " $X$ is homeomorphic to a subspace of $j(X)$ " by " $X$ is a perfect image of a subspace of $j(X)$ ". By definition, topological properties are preserved by homeomorphisms; many topological properties are also perfect invariants, i.e. preserved both directly and inversely by perfect maps.

The exposition in [GJT98] is good, so we will confine ourself to just saying a few words about it. The key observation is that much of the reflection technology still works in this perfect image situation. In particular, one can use the perfect map to lift a discrete collection in $X$ (which is homeomorphic to $j$ " $X$ ) to $j(X)$, use normality to separate it there, since the size of its union is $<j(X)$, and then use the perfect map to bring down the separation to $j$ " $X$. To obtain the perfect map, send $\bigcap\{j$ " $V: V$ is open, $x \in V\}$ to $j(x)$. The use of local compactness is to prove that this intersection equals $\bigcap\left\{j\right.$ " $K: K$ is a compact $\left.G_{\delta}, x \in K\right\}$. These observations about perfect maps were generalized in [JT98] to the topology of elementary submodels context (see Section 6). "Generalized" because $j$ " $H_{\theta}$ is an elementary submodel of $j\left(H_{\theta}\right)=H_{j(\theta)}$.

The proof that locally compact normal spaces are collectionwise normal in this model extends naturally to replace locally compact by pointwise countable type. A space has pointwise countable type if every point is contained in a compact set of countable character. The largest class of naturally defined spaces for which one could hope to consistently prove that normality implies collectionwise normality are the $k$-spaces, i.e. spaces in which a set is closed if and only if its intersection with every compact set is compact. I conjecture that this can be done, and the way to do it is to induct on the $k$-order of the spaces. $k$-order was defined by Arhangel'skil in [Arh65] in analogy to sequential order. The first step in the program was accomplished by Renata Prado [Pra99] in her Ph.D thesis, using a weakening of "perfect map" to adapt the pointwise countable type proof to the class of $k^{\prime}$-spaces, i.e. spaces such that whenever $x \in \bar{F}, F \subseteq X$, then there is a compact $K \subseteq X$ such that $x \in \overline{F \cap K}$.

The consistency of normality implying collectionwise normality for $k^{\prime}$-spaces had been shown earlier by Daniels [Dan91], using the Product Measure Extension Axiom, but there was no obvious way to continue along that path.

Having seen the use of preservation arguments for Cohen and random forcing in reflection proofs, we are encouraged to examine other classes of partial orders with a view toward preservation. Where we would really like to be able to apply preservation is in the case of countably closed forcing, for then we could get reflection after Lévy-collapsing a supercompact to $\aleph_{2}$. Unfortunately, not much is known about this. We would like to show that Lindelöfness is preserved for $T_{2}$ spaces with points $G_{\delta}$; instead, as we saw above, the stronger Rothberger property is preserved. We would like to show that non-collectionwise Hausdorff is preserved; instead Shelah 
[She77] showed this was for locally separable, first countable spaces. Solving either of these questions affirmatively would lead to the solution of long-outstanding open problems: the first, a consistency proof for "every Lindelöf $T_{2}$ space with points $G_{\delta}$ has cardinality $\leq 2^{\aleph_{0}}=\aleph_{1}$ " [Tal95]; the second, a consistency proof for "every first countable $\aleph_{1}$-collectionwise Hausdorff space is collectionwise Hausdorff" [Tal07].

\section{TOPOLOGY OF ELEMENTARY SUBMODELS}

Once one introduces a new mathematical technique for the purpose of attacking certain problems, the technique itself often becomes an object of study. One such technique is that of elementary submodels. The best published exposition of the basic facts for set theorists is Chapter 24 of [JW97], which we shall assume the reader is acquainted with. A large number of topological applications are in [Dow88]. The first paper to systematically study the technique itself is [JT98]. There, given a topological space $\langle X, \mathcal{J}\rangle$ in an elementary submodel $M$ of a sufficiently large $H_{\theta}, \theta$ regular, we define $X_{M}$ to be $X \cap M$ with topology $\mathcal{J}_{M}$ generated by $\{U \cap M: U \in \mathcal{J} \cap M\}$. For the student not so familiar with elementary submodels, let us note that the incantation about $H_{\theta}$ is chanted in order to avoid dealing with $M$ 's which are elementary submodels of $V$, because there are Gödelian difficulties in doing do. For all practical purposes, we think of $M$ as indeed being an elementary submodel of $V$. For elucidation of this point, see the chapter of [JW97] referred to above.

There are two natural questions associated with the study of $X_{M}$ :

(1) Given $X$ and $M$, what does $X_{M}$ look like?

(2) Given $X_{M}$, what does $X$ look like? In particular, if $X_{M}$ is homeomorphic to some nice space, must $X_{M}=X$ ?

$X_{M}$ is not the only way of forming a new space, given $X$ and $M$. See e.g. Eisworth [Eis06] and Section 3.5 of [Pra99].

Question 1 is pretty well dealt with in [JT98], so we will not get into it here. However, one point I want to call attention to is that - in contrast to the abundance of examples of different topological spaces with various combinations of properties - at present there seem to be only a few different kinds of elementary submodels. Perhaps this is a good subject for further study.

Even powers of the two-point discrete space $D$ reveal interesting questions about Question 2: if $X_{M}$ is homeomorphic to $D^{\kappa}$, does $X=X_{M}$ ? In a series of papers, [Tal00b], [JT03], [Kun03], [Tal04], [JLT06], [Tal06], it transpired that the answer is positive for small cardinals, but can be negative for large ones. Moreover, the essential property of $D$ turned out to be that it satisfies the countable chain condition. Some sample results are:

Proposition 6.1 ([Kun03]). Let $\kappa$ be the first supercompact. Let $X$ be a compact space of size $\geq \kappa$. Then for some elementary submodel $M$ containing $X, X_{M}$ is compact and not equal to $X$.

Proposition 6.2 ([Tal06]). If $X_{M}$ is compact and satisfies the countable chain condition, and if $X$ is not scattered and $|X|<$ the first inaccessible, then $X=X_{M}$. If the character of $X_{M}$ is in $M$ then "inaccessible" can be replaced by "1-extendible".

There remains one intriguing problem I wish to bring to the reader's attention:

Problem 6.3. If $X_{M}$ is homeomorphic to the space of irrationals, does $X_{M}=X$ ? 
This is still open; partial results were achieved in [Tal02].

This question seems natural; for such familiar spaces as $\mathbb{R}$ (the real line), $\mathbb{Q}$ (the rationals), $\mathbb{P}$ (the irrationals), $\mathbb{K}$ (the Cantor set), we ask whether if $X_{M}$ is homeomorphic to any of these, must $X=X_{M}$ ?

For $\mathbb{Q}$, there is a simple negative answer. Let $X$ be any $T_{3}$ space without isolated points. Suppose $M$ is a countable elementary submodel of $H_{\theta}$, for $\theta$ a sufficiently large regular cardinal. Then $X_{M}$ is homeomorphic to $\mathbb{Q}$. The reason is that $X_{M}$ is a countable $T_{3}$ space with a countable base and with no isolated points. All such spaces are homeomorphic to $\mathbb{Q}[$ Eng89, Exercise 6.2.A(d)].

Theorem 6.4 ([Tal00b], [Tal02]). If $X_{M}$ is a locally compact uncountable separable metrizable space, then $X=X_{M}$.

Proof. We first prove it suffices to show that $[0,1] \subseteq M$ - any definable space of power $2^{\aleph_{0}}$ would do. It follows that $\omega_{1} \subseteq M$. Now first observe that if $X_{M}$ is $T_{2}$, so is $X$. Then recall that $\left\{x_{\alpha}\right\}_{\alpha<\omega_{1}}$ is left- (right-) separated if there exist open sets $\left\{U_{\alpha}: \alpha<\omega_{1}\right\}$, with $\alpha \in U_{\alpha}$ such that $U_{\alpha} \cap\left\{x_{\beta}: \beta<\alpha\right\}\left(\left\{x_{\beta}: \beta>\alpha\right\}\right)=0$. If $\omega_{1} \subseteq M$ and $X$ has an uncountable left- or right-separated subspace, so does $X_{M}$ - simply relativize the assertion that there is such a subspace to $M$. Separable metrizable spaces don't have uncountable left- or right-separated subspaces since they are hereditarily separable and hereditarily Lindelöf, so $X$ doesn't. $X_{M}$ is also $T_{3}$. It is a standard cardinal function exercise [Juh71], [Roi84] to show that the nonexistence of an uncountable left-separated subspace implies a space is hereditarily separable and therefore (by $T_{3}$ ) has weight $\leq 2^{\aleph_{0}}$, and that the non-existence of an uncountable right-separated subspace implies a space is hereditarily Lindelöf, and hence, if its weight is $\leq 2^{\aleph_{0}}$, has no more than $2^{\aleph_{0}}$ open sets. But if $[0,1] \subseteq M$ and both $X$ and its topology $\mathcal{J}$ have cardinality $\leq 2^{\aleph_{0}}$, then $X$ and $\mathcal{J}$ are included in $M$, so $X=X_{M}$.

Now, to show $[0,1] \subseteq M$, take a basic open subset $V=U \cap M, U$ open in $X$, $U \in M$, of $X_{M}$, with compact closure. Then $\bar{U} \in M$. Observe that $\bar{V}=(\bar{U})_{M}$; that $(\bar{U})_{M}=\bar{U} \cap M \subseteq \bar{V}$ is clear, if $x \in \bar{V}$ were not in $\bar{U}$, there would be a $W$ open in $X_{M}$ containing $x$ such that $W \cap U=0$. Take such a $W \in M$. Then $W \cap M \cap U=0$, contradiction.

We need a result of Lucia Junqueira [Jun00]:

Lemma 6.5. If $Y_{M}$ is compact, so is $Y$, and, in fact, $Y_{M}$ is a perfect image of $Y$.

Then $\bar{U}$ maps perfectly onto $(\bar{U})_{M} \cdot \bar{U}$ includes a copy of the Cantor set, so a closed subspace of $\bar{U}-$ and hence, by the Tietze Extension Theorem $-\bar{U}$ itself maps onto $[0,1]_{M}=[0,1] \cap M$ (by the first countability of $[0,1]$ ). Since $[0,1]$ is separable, $[0,1] \cap M$ is dense in $[0,1]$. But $[0,1] \cap M$ is compact, so $[0,1] \cap M=[0,1]$, i.e. $[0,1] \subseteq M$.

For the Irrational Problem, the basic difficulty is that, unlike compactness, completeness - as far as I know - does not "lift" from $X_{M}$ to $X$. There are a variety of disparate sufficient conditions that ensure that $X_{M}=X$. They all aim to either ensure that $X$ includes a copy of the Cantor set, or else ensure that $M$ is sufficiently "fat", so that if its cardinality is $\leq 2^{\aleph_{0}}$, it actually includes $2^{\aleph_{0}}$ and hence $M$. The question of whether elementary submodels of size $\geq 2^{\aleph_{0}}$ (entailed e.g. by $X_{M}$ being uncountable, separable, and completely metrizable) must necessarily include $2^{\aleph_{0}}$ is an intriguing one. In [KT00], we proved this follows from the non-existence of $0^{\#}$. 
Welch [Wel02] noted this also follows from the assumption that $2^{\aleph_{0}}$ is not a Jónsson cardinal. On the other hand, Chang's Conjecture plus the Continuum Hypothesis implies there is an elementary submodel $M$ of size $2^{\aleph_{0}}$ with $2^{\aleph_{0}} \cap M$ countable [Tal00a].

Theorem 6.6. Suppose $X_{M}$ is an uncountable, separable, completely metrizable space. Then $X=X_{M}$ if any of the following conditions hold:

a) $X$ includes a copy of the Cantor set,

b) $X$ is of pointwise countable type,

c) $|\mathbb{R} \cap M|$ is uncountable,

d) $|X|=2^{\aleph_{0}}$.

Clause a) begs a question I do not know the answer to:

Problem 6.7. If $X_{M}$ includes a copy of a Cantor set, does $X$ ?

The proof of a) follows along the same lines as that of Theorem 6.4. We again wish to obtain a closed map from a closed - hence complete - subspace of $X_{M}$ onto $[0,1]_{M}=[0,1] \cap M$. Closed metrizable images of completely metrizable spaces are completely metrizable [Eng89, 4.4.17] and hence absolute $G_{\delta}$. If $[0,1] \cap M$ is a $G_{\delta}$ in $[0,1]$, it is all of $[0,1]$ by $[\mathrm{Kec} 95,0.11]$, and now we can finish as before. Unfortunately, just because there is a closed map from a closed subspace of $X$ onto $[0,1]$ does not mean there is a closed map from a closed subspace of $X_{M}$ onto $[0,1] \cap M$. The difficulty is that elementarity will tell us about basic closed subsets of $X_{M}$, but not necessarily about arbitrary closed sets. Nonetheless, one can push this idea for a proof through — see [Tal02]. As part of the proof, we use maps that behave nicely with respect to basic closed sets, but - unlike continuous maps need not necessarily then behave nicely with respect to all closed sets. This topic deserves further study.

Clause b) follows from:

Lemma 6.8 ([JT98]). If $X$ is of pointwise countable type, then $X_{M}$ is a perfect image of a subspace of $X$.

Clause c) follows from a theorem of Woodin [Tal02]:

Lemma 6.9. If $\mathbb{R} \cap M$ includes a perfect set, then $\mathbb{R} \cap M=\mathbb{R}$.

We get a continuous map from a $G_{\delta}$ subspace of $X_{M}$ onto $[0,1] \cap M$ and thus get $\mathbb{R} \cap M$ analytic. But an analytic uncountable set of reals includes a perfect set.

Clause d) follows directly from c), and is left for the reader.

\section{LINDELÖF PROBLEMS}

There are quite a few classical problems involving Lindelöf spaces. Aspiring settheoretic topologists could pick any one of these as a thesis problem. My philosophy with regards to thesis problems is that one should work on something significant. Even if one doesn't solve it, one will likely amass sufficiently many related results so as to have a thesis. I consider this more worthwhile than just solving some insignificant problem one's supervisor already knows how to solve.

Here are some sample Lindelöf problems I have been looking at:

Problem 7.1. Is there a Lindelöf space which is not a D-space? A space is a $D$-space if for each funtion $f$ assigning to each $x \in X$ a neighbourhood $N(x)$, there is a closed discrete $D$ such that $\bigcup\{N(x): x \in D\}$ covers $X$. 
For this problem, consult [Gru] in this volume.

Problem 7.2. Find a reasonable bound on the Lindelöf number of a product of two Lindelöf spaces. Maybe $2^{2^{\aleph_{0}}}$ will do, or perhaps $2^{\aleph_{0}}$ consistently. It is consistent that the product can have Lindelöf number $>2^{\aleph_{0}}$ - see [Juh84] and [Gor94]. The only absolute bound known is the first strongly compact cardinal, which is far too large. To obtain that bound, mindlessly generalize the Tychonoff Theorem.

Problem 7.3. Call a space productively Lindelöf if it is Lindelöf and its product with every Lindelöf space is Lindelöf. Are productively Lindelöf metrizable spaces always $\sigma$-compact? Indeed it is not hard to see that $\sigma$-compact spaces are productively Lindelöf. This problem is a variant of E. Michael's classic problem as to whether there is a Lindelöf space whose product with the space of irrationals is not normal (such a space is called a Michael space). The latter problem has numerous connections with cardinal invariants of the continuum — see for example [Moo99].

Set theory is constantly rearing its playful head in the kind of topology I do; the latest example is:

Theorem 7.4 ([Tal09]). The Axiom of Projective Determinacy implies that every productively Lindelöf projective set of reals is $\sigma$-compact if and only if there is a Michael space.

There are connections between Problems 7.1 and 7.3. These are briefly surveyed in [Gru]. For details, see [Aur10], [AT], [Tal09], [Tal10a], [Tal10b]. The most interesting recent result on the Lindelöf $D$ problem is due to Leandro Aurichi [Aur10]. The classical Menger property is like the Rothberger property defined above, except picking a finite subset rather than a singleton from each one of the countable sequence of open covers.

Proposition 7.5 ([Aur10]). Every Menger space is D.

From this we obtain:

Proposition 7.6 ([Tal10b]). The Continuum Hypothesis implies that if the product of $X$ with every Lindelöf space is Lindelöf, then $X$ is a D-space.

We survey these and other Lindelöf problems in [Tal11b]

In conclusion, let me thank for their attention the graduate students and postdocs who attended the course that led to this survey. I also thank the referee for helpful comments.

\section{REFERENCES}

[Arh65] A. V. Arhangel'skiǔ, Bicompact sets and the topology of spaces, Trans. Moscow Math. Soc. 13 (1965), 1-62. MR 0195046 (33 \#325)

[Arh72] _ The property of paracompactness in the class of perfectly normal locally bicompact spaces, Dokl. Akad. Nauk SSSR 203 (1972), 1231-1234. MR 0305342 (46 \#4472)

[AT] L. F. Aurichi and F. D. Tall, Lindelöf spaces which are productive, Hurewicz, or D, submitted.

[AT97] U. Abraham and S. Todorcevic, Partition properties of $\omega_{1}$ compatible with $C H$, Fund. Math. 152 (1997), 165-181. MR 1441232 (98b;03064)

[Aur10] L. F. Aurichi, D-spaces, topological games and selection principles, Topology Proc. 36 (2010), 107-122. 
[Bal83] Z. T. Balogh, Locally nice spaces under Martin's axiom, Comment. Math. Univ. Carolin. 24 (1983), no. 1, 63-87. MR 703926 (85b:54005)

[Bal91] , On the collectionwise normality of locally compact normal spaces, Trans. Amer. Math. Soc. 323 (1991), 389-411. MR 098571 (91c;54030)

[Bal02] L Locally nice spaces and axiom R, Topology Appl. 125 (2002), no. 2, 335-341. MR 1933581 (2003i:54029)

[Bel81] M. Bell, On the combinatorial principle $P(c)$, Fund. Math. 14 (1981), 149-157. MR 0642449 (82m;03064)

[BT02] J. E. Baumgartner and F. D. Tall, Reflecting Lindelöfness, Proceedings of the International Conference on Topology and its Applications (Yokohama, 1999), vol. 122, Yokohama 1999, no. 1-2, 2002, pp. 35-49. MR 1919290 (2003m:54027)

[Dan91] P. Daniels, Normal $k^{\prime}$ spaces are consistently collectionwise normal, Fund. Math. 138 (1991), 225-234. MR 1121609 (92g;54031)

[Dev83] K. J. Devlin, The Yorkshireman's guide to proper forcing, Surveys in set theory (A.R.D. Mathias, ed.), London Math. Soc. Lecture Note Ser., vol. 87, Cambridge Univ. Press, Cambridge, 1983, pp. 60-115. MR 823776 (87h:03081)

[Dow88] A. Dow, An introduction to applications of elementary submodels in topology, Top. Proc. 13 (1988), no. 1, 17-72. MR 1031969 (91a;54003)

[Dow92] Set theory in topology, Recent progress in general topology (Prague, 1991) (M. Hušek and J. van Mill, eds.), North-Holland, Amsterdam, 1992, pp. 167-197. MR 1229125

[DS79] K. J. Devlin and S. Shelah, A note on the normal Moore space conjecture, Canad. J. Math. 31 (1979), 241-251. MR 05028801 (81d;54022)

[DTW90a] A. Dow, F. D. Tall, and W. A. R. Weiss, New proofs of the consistency of the normal Moore space conjecture. I, Topology Appl. 37 (1990), no. 1, 33-51. MR 1075372 (92b:54008a)

[DTW90b] A. Dow, F. D. Tall, and W. A. R. Weiss, New proofs of the consistency of the normal Moore space conjecture. II, Topology Appl. 37 (1990), no. 2, 115-129. MR 1080345 (92b:54008b)

[Eas70] W.B. Easton, Powers of regular cardinals, Ann. Math. Logic 1 (1970), 129-178. MR 0269497 (42 \#4392)

[Eis06] T. Eisworth, Elementary submodels and separable monotonically normal compacta, Top. Proc. 30 (2006), no. 2, 431-443. MR 2352742 (2008954039)

[EN09] T. Eisworth and P. J. Nyikos, Antidiamond principles and topological applications, Trans. Amer. Math. Soc. 361 (2009), 5695-5719. MR 2529910

[Eng89] R. Engelking, General Topology, Heldermann Verlag, Berlin, 1989. MR 1039321 (91c:54001)

[Far96] I. Farah, OCA and towers in $\mathcal{P}(\mathbb{N}) /$ Fin, Comment. Math. Univ. Carolin. 37 (1996), 861-866. MR 1440716 (98f:03043)

[Fis] A. Fischer, Ph.D. thesis, University of Toronto, in preparation.

[Fle74] W. G. Fleissner, Normal Moore spaces in the constructible universe, Proc. Amer. Math. Soc. 62 (1974), 294-298. MR 0362240 (50 \#14682)

[Fle78] Separation properties in Moore spaces, Fund. Math. 98 (1978), 275-286. MR 0521125 (80f:54009)

[Fle86] _ Left separated spaces with point-countable bases, Trans. Amer. Math. Soc. 294 (1986), no. 2, 665-677. MR 825729 (87d:03134)

[GJT98] R. Grunberg, L.R. Junqueira, and F.D. Tall, Forcing and normality, Topology Appl. 84 (1998), 145-174. MR 1611214 (99e:54016)

[GK96a] G. Gruenhage and P. Koszmider, The Arkhangel'skiu-Tall problem: a consistent counterexample, Fund. Math. 13 (1996), 143-166. MR 1383211 (97g:54004)

[GK96b] _ The Arkhangel'skiü-Tall problem under Martin's axiom, Fund. Math. 149 (1996), no. 3, 275-285. MR 1383211 (97g:54004)

[Gor94] I. Gorelic, On powers of Lindelöf spaces, Comment. Math. Univ. Carolin. 35 (1994), no. 2, 383-401. MR 1286586 (95e:54009)

[Gru] G. Gruenhage, A survey of D-spaces, this volume.

[HJ76] A. Hajnal and I. Juhász, Remarks on the cardinality of compact spaces and their Lindelöf subspaces, Proc. Amer. Math. Soc. 59 (1976), no. 1, 146-148. MR 0423283 (54 \#11263) 
[Iwa07] A. Iwasa, Covering properties and Cohen forcing, Top. Proc. 31 (2007), 553-559. MR 2476629 (2009j:54033)

[JLT06] L. R. Junqueira, P. Larson, and F. D. Tall, Compact spaces, elementary submodels, and the countable chain condition, Ann. Pure and Applied Logic 144 (2006), 107-116. MR 2279657 (2007j:54005)

[JT98] L. R. Junqueira and F. D. Tall, The topology of elementary submodels, Topology Appl. 82 (1998), 239-266. MR 1602479 (99a:54003)

[JT03] L.R. Junqueira and F. D. Tall, More reflections on compactness, Fund. Math. 176 (2003), 127-141.

[Juh71] I. Juhász, Cardinal functions in topology, Math Centrum, Amsterdam, 1971. MR 0340021 (49 \#4778)

[Juh84] Cardinal functions. II, Handbook of Set-theoretic Topology (K. Kunen and J. E. Vaughan, eds.), North-Holland, Amsterdam, 1984, pp. 63-109. MR 776621 (86j:54008)

[Jun00] L. R. Junqueira, Upwards preservation by elementary submodels, Topology Proc. 25 (2000), 225-249. MR 1875594 (2003b:03047)

[JW97] W. Just and M. Weese, Discovering Modern Set Theory, II, Amer. Math. Soc., Providence, 1997. MR 1474727 (99b:03001)

[Kad] M. Kada, Remarks on the preservation of topological covering properties under Cohen forcing, preprint.

[Kan94] A. Kanamori, The Higher Infinite, Springer-Verlag, Berlin, 1994. MR 1321144 (96k:03125)

[Kat48] M. Katětov, Complete normality of Cartesian products, Fund. Math. 35 (1948), 271274. MR $0027501(10,315 \mathrm{~h})$

[Kec95] A. S. Kechris, Classical Descriptive Set Theory, Springer-Verlag, New York, 1995. MR 1321597 (96e:03057)

[KM78] A. Kanamori and M. Magidor, The evolution of large cardinal axioms in set theory, Lect. Notes. Math. 669, Springer-Verlag, New York, 1978, pp. 99-275. MR 0520190 (80b:03083)

[KT79] K. Kunen and F. D. Tall, Between Martin's axiom and Souslin's hypothesis, Fund. Math. 102 (1979), no. 3, 173-181. MR 532951 (83e:03078)

[KT00] _ The real line in elementary submodels of set theory, J. Symbolic Logic 65 (2000), 683-691. MR 1771077 (2002f:03071)

[KT02] P. Koszmider and F. D. Tall, A Lindelöf space with no Lindelöf subspace of size $\aleph_{1}$, Proc. Amer. Math. Soc. 130 (2002), no. 9, 2777-2787. MR 1900885 (2003a:54002)

[Kun80] K. Kunen, Set theory, North-Holland, Amsterdam, 1980. MR 0597342 (82f:03001)

[Kun03] Compact spaces, compact cardinals, and elementary submodels, Topology Appl. 130 (2003), 99-109. MR 1973391 (2004b:54037)

[Lar99] P. Larson, An $\mathbb{S}_{\max }$ variation for one Souslin tree, J. Symbolic Logic 64 (1999), no. 1, 81-98. MR 1683897 (2000g:03118)

[Lav78] R. Laver, Making the supercompactness of $\kappa$ indestructible under $\kappa$-directed closed forcing, Israel J. Math. 29 (1978), no. 4, 385-388. MR 0472529 (57 \#12226)

[LTaa] P. Larson and F. D. Tall, Locally compact perfectly normal spaces may all be paracompact, Fund. Math., to appear.

[LTab] _ On the hereditary paracompactness of locally compact hereditarily normal spaces, preprint.

[LTo01] P. Larson and S. Todorcevic, Chain conditions in maximal models, Fund. Math. 168 (2001), 77-104. MR 1835483 (2002e:03067)

[LTo02] Katětov's problem, Trans. Amer. Math. Soc. 354 (2002), no. 5, 1783-1791. MR 1881016 (2003b:54033)

[Moo99] J. T. Moore, Some of the combinatorics related to Michael's problem, Proc. Amer. Math. Soc. 127 (1999), 2459-2467. MR 1486743 (99j:54008)

[Nyi83] P. J. Nyikos, Set-theoretic topology of manifolds, General topology and its relations to modern analysis and algebra, V (Prague, 1981), Sigma Ser. Pure Math., vol. 3, Heldermann, Berlin, 1983, pp. 513-526. MR 698452 (85i:54004)

[Nyi84] The theory of nonmetrizable manifolds, Handbook of Set-theoretic Topology (K. Kunen and J. E. Vaughan, eds.), North-Holland, Amsterdam, 1984, pp. 633-684. MR 0776633 (86f:54054) 
[Nyi02] Complete normality and metrization theory of manifolds, Proceedings of the Janós Bolyai Mathematical Society 8th International Topology Conference (Gyula, 1998), vol. 123, 2002, pp. 181-192. MR 1921659 (2003f:54048)

[Nyi03] Applications of some strong set-theoretic axioms to locally compact $T_{5}$ and hereditarily scwH spaces, Fund. Math. 176 (2003), no. 1, 25-45. MR 1971471 (2004k:54008)

[Paw94] J. Pawlklowski, Undetermined sets of point-open games, Fund. Math. 144 (1994), 279-285. MR 1279482 (95i:54043)

[Pra99] R. G. A. Prado, Applications of Reflection to Topology, Ph.D. thesis, University of Toronto, 1999.

[Roi84] J. Roitman, Basic $S$ and L, Handbook of Set-Theoretic Topology (K. Kunen and J. E. Vaughan, eds.), North-Holland, Amsterdam, 1984, pp. 295-326. MR 776626 (87a:54043)

[Sch] M. Scheepers, Measurable cardinals and the cardinality of Lindelöf spaces, submitted.

[She77] S. Shelah, Remarks on $\lambda$-collectionwise Hausdorff spaces, Proceedings of the 1977 Topology Conference (Louisiana State Univ., Baton Rouge, La., 1977), II, vol. 2, 1977, pp. 583-592 (1978). MR 540629 (81f:03060)

[She96] Remarks on $\aleph_{1}-C W H$ not $C W H$ first countable spaces, Set theory (Boise, ID, 1992-1994), Contemp. Math., vol. 192, Amer. Math. Soc., Providence, RI, 1996, pp. 103-145. MR 1367139 (96m:54008)

[ST] M. Scheepers and F.D. Tall, Lindelöf indestructibility, topological games and selection principles, Fund. Math., to appear.

[Tal74] F. D. Tall, On the existence of normal metacompact Moore spaces which are not metrizable, Canad. J. Math. 26 (1974), 1-6. MR 0377823 (51 \#13992)

[Tal77]_, Set-theoretic consistency results and topological theorems concerning the normal Moore space conjecture and related problems. Doctoral Dissertation, University of Wisconsin (Madison), 1969; Dissertationes Math. (Rozprawy Mat.) 148 (1977), 53. MR 0454913 (56 \#13156)

[Tal84] Normality versus collectionwise normality, Handbook of Set-theoretic Topology (K. Kunen and J. E. Vaughan, eds.), North-Holland, Amsterdam, 1984, pp. 685732. MR 776634 (86m:54022)

[Tal88]__ Covering and separation properties in the Easton model, Topology Appl. 28 (1988), no. 2, 155-163. MR 932980 (89e:54008)

[Tal94a] $\quad \sigma$-centred forcing and reflection of (sub)metrizability, Proc. Amer. Math. Soc. 121 (1994), 299-306. MR 1179593 (94g:54003)

[Tal94b] Topological applications of generic huge embeddings, Trans. Amer. Math. Soc. 341 (1994), no. 1, 45-68. MR 1223302 (94j:03108)

[Tal95] _ On the cardinality of Lindelöf spaces with points $G_{\delta}$, Topology Appl. 63 (1995), no. 1, 21-38. MR 1328616 (96i:54016)

[Tal00a] , If it looks and smells like the reals..., Fund. Math. 163 (2000), 1-11. MR 1750331 (2001d:54003)

[Tal00b] L Reflecting on compact spaces, Top. Proc. 25 (2000), 345-350. MR 1925692 (2003i:54008)

[Tal02] $\quad$ An irrational problem, Fund. Math. 175 (2002), 259-269. MR 1969659 (2004c:54012)

[Tal04] $\quad$ Reflections on dyadic compacta, Topology Appl. 137 (2004), 251-258. MR 2057891 (2005a:54004)

[Tal06] Compact spaces, elementary submodels and the countable chain condition, II, Topology Appl. 153 (2006), 273-278. MR 2243743 (2007j:54006)

[Tal07] , Reflection of topological properties to $\aleph_{1}$, Open problems in topology, II (E. Pearl, ed.), Elsevier, New York, 2007, pp. 241-247.

[Tal09] Lindelöf spaces which are Menger, Hurewicz, Alster, productive, or D, Topology Appl., to appear, 2009.

[Tal10a] - A note on productively Lindelöf spaces, submitted, 2010.

[Tal10b] - Productively Lindelöf spaces may all be D, submitted, 2010.

[Tal11a] Characterizing paracompactness in locally compact normal spaces, preprint, 2011.

[Tal11b] , Set-theoretic problems concerning Lindelöf spaces, preprint, 2011. 
[Tal11c] _ PFA(S)[S] and the Arhangel'skiǔ-Tall problem, in preparation, 2011.

[Tay81] A. D. Taylor, Diamond principles, ideals and the normal Moore space problem, Canad. J. Math. 33 (1981), no. 2, 282-296. MR 617619 (83a:03049)

[Tod] S. Todorcevic, Chain conditions in topology, II, in preparation.

[Tod08]_ Handwritten slides from his lectures at Advances in Set-Theoretic Topology, a Conference in Honor of T. Nogura, Erice, Italy, 2008.

[Wat82] W. S. Watson, Locally compact normal spaces in the constructible universe, Canad. J. Math. 34 (1982), no. 5, 1091-1096. MR 675681 (83k:54021)

[Wei84] W. A. R. Weiss, Versions of Martin's Axiom, Handbook of Set-Theoretic Topology (K. Kunen and J. E. Vaughan, eds.), North-Holland, Amsterdam, 1984, pp. 827-886. MR 0776638 (86h:03088)

[Wel02] P. D. Welch, On possible non-homeomorphic substructures of the real line, Proc. Amer. Math. Soc. 130 (2002), 2771-2775. MR 1900884 (2003h:03083)

Department of Mathematics, University of Toronto, Toronto, Ontario, M5S 2E4, Canada

E-mail address: f.tall@utoronto.ca 